\title{
Variability of Non-Schmid Effects in Grain Boundary Dislocation Nucleation Criteria
}

Richard D Wyman ${ }^{1}$, David T Fullwood ${ }^{1}$, Robert H Wagoner ${ }^{2}$, Eric R Homer ${ }^{1^{*}}$

1 Department of Mechanical Engineering, Brigham Young University, Provo, UT, USA.

2 Department of Materials Science and Engineering, Ohio State University, Columbus, OH, USA

\begin{abstract}
Grain boundary dislocation nucleation is simulated in a Nickel bicrystal with $\Sigma 21$ b (211)/(211) grain boundaries. Using molecular dynamics, 386 different triaxial stress states are examined for their effect on dislocation nucleation on different slip systems. The approach leads to dislocation nucleation on six of the twelve partial slip systems, enabling a study of conditions leading to nucleation. The criteria for nucleation on each of the slip systems is shown to have a linear dependence on the resolved shear, normal, and co-slip stresses, though the constants for this linear dependence are unique for each slip system. The combined nucleation criteria are used to construct a theoretical nucleation surface. The surface is reminiscent of a MohrCoulomb yield surface, except that in the present case the facets of the surface correspond to nucleation on different slip systems.
\end{abstract}

Keywords:

Grain Boundaries, Dislocation Nucleation, Molecular Dynamics

* Corresponding author: eric.homer@byu.edu 


\section{Introduction}

Grain boundaries (GBs) play an important role in material strength, becoming increasingly important as grain size shrinks [1-4]. GBs act as barriers to dislocation motion and as sources and sinks for dislocations. Of primary interest in this work is the heterogeneous nucleation of dislocations, where a GB relieves local stress by emitting dislocations in one or both of the grains it separates [5-7].

Similar to homogeneous dislocation nucleation in the absence of a GB [8-10], nucleation at a GB is known to have non-Schmid stress dependence $[5,11]$. In particular, the critical resolved shear stress for dislocation nucleation is heavily dependent upon the resolved normal stress, where the normal stress acts perpendicular to the slip plane, as shown schematically in Figure 1. When the resolved normal stress is compressive, it is harder for atoms to glide over each other, but when it is tensile, it is easier for atoms to glide [5].

Investigations into the conditions required for dislocation nucleation have led to a number of different conclusions. Spearot, et al. applied uniaxial tension to a number of $\langle 100\rangle$ and $\langle 110\rangle$ tilt GBs [5]. By using a least squares fit, they built an equation to predict the uniaxial stress required to cause GB dislocation nucleation. They found that dislocation nucleation from $\langle 100\rangle$ tilt GBs is governed primarily by the resolved shear stress while $\langle 110\rangle$ tilt GBs are governed primarily by resolved normal stress. Beyerlein, et al. proposed that dislocation nucleation is favored on slip systems well aligned with intrinsic dislocations inside the GB. The likelihood of nucleation on some slip system is then proportional to the driving stress multiplied by a structure factor quantifying the favorability of some slip systems [6]. Sangid, et al. developed a method of measuring energy barriers to dislocation nucleation and found that the energy barrier for nucleation is inversely proportional to the static GB energy [12], and the nucleation event leads to a drop in GB interfacial energy $[12,13]$. Additional work by Spearot et al. found increased nanoporosity in a GB leads to nucleation at relatively low stresses [14], and Wang et al. found that high creep stresses can induce dislocation nucleation from GBs in nanocrystalline materials [15]. The current consensus is that GB dislocation nucleation is tied to GB structure and that resolved shear alone cannot predict when nucleation will occur. 
Other factors surrounding the location of GB dislocation nucleation have also received attention. Wu, et al. show that the point of nucleation within a GB is correlated with high Von Mises stress [16]. Similarly, Zhang, et al. show that nucleation tends to occur at locations with the most intense localized shear [17]. Burbery, et al. correlate the nucleation location with atoms whose virial stress has a large component normal to the $G B$, and also note that per atom potential energy fails to predict the location of nucleation [18]. None of these observations are exclusive of the others, as all suggest nucleation occurs at locations of high stress; however, they also do not provide an overall theory on where and at what stress nucleation will occur. Such predictive capability remains an elusive goal.

In the present work, we examine a single GB under many different triaxial stress states. The approach is designed to investigate how different stress states influence dislocation nucleation on different slip systems. Using stress states measured near the GB at the time nucleation occurs, we are able to determine the relative influence of shear, normal, and co-slip stresses on GB dislocation nucleation. The analysis produces unique nucleation criteria for different slip systems that can combine to give a nucleation surface that predicts dislocation nucleation. The significance of these results are discussed in the context of the literature.

\section{Methodology}

\subsection{Molecular Dynamics Simulations}

The present work simulates an atomistic model of a $\Sigma 21 \mathrm{~b}$ [211] $44.415^{\circ}$ symmetric twist Nickel GB subjected to a range of triaxial stress states to measure the conditions surrounding GB dislocation nucleation. The GB has (211) boundary planes in both crystals and can also be described as a [421] $180^{\circ}$ symmetric tilt GB. The work focuses on one of two GBs in a fully periodic bicrystal. The bicrystal is shown in Figure 2 with atoms colored by their common neighbor analysis (CNA) value [19]. The smaller middle grain, grain A, is $126 \AA$ wide, the larger outer grain, grain B, is $234 \AA$ wide. The dimensions in the $y$ and $z$ directions of the orthogonal

simulation cell are $33 \AA$ and $32 \AA$, respectively. In total, the system contains 31,500 atoms. The bicrystal is derived from one of the computed GBs prepared by Olmsted, et al. [20,21]. Molecular dynamics is performed with LAMMPS [22] utilizing GPU accelerated hardware [23- 
25] with the Foiles-Hoyt Nickel embedded atom potential [26]. Visualization of the bicrystal is performed with OVITO [27]. The bicrystal is simulated at 0.1 Kelvin to minimize the contributions of thermal energy, as has been done in other work [11]. The bicrystal is equilibrated at the desired temperature and zero pressure for 100 ps using an NPT ensemble and the Nosé-Hoover thermostat $[28,29]$.

To achieve the range of desired triaxial stress states to be applied to the simulation cell, each individual stress state is specified by a unit vector, $\lambda$. Each component of this $\lambda$ vector represents the relative stress magnitude and sign in the $\hat{x}, \hat{y}$, and $\hat{z}$ dimensions, respectively. The $\lambda$ vector is then defined as

$$
\lambda=\frac{\left[\sigma_{x}, \sigma_{y}, \sigma_{z}\right]}{\sqrt{\sigma_{x}^{2}+\sigma_{y}^{2}+\sigma_{z}^{2}}}
$$

To examine the full range of triaxial stress states, we select 386 unique points approximately equidistributed about a unit sphere [30] where the Cartesian coordinates of each point on the sphere gives the values for the $\lambda$ unit vectors.

For each $\lambda$ vector, molecular dynamics is used to simulate a triaxial stress test on the bicrystal with the intention of inducing GB dislocation nucleation. The Nosé-Hoover thermostat is again used to maintain the desired temperature and ramp the applied $\sigma_{x}, \sigma_{y}$, and $\sigma_{z}$ stresses in their corresponding directions from 0 GPa such that their geometric norm grows at a rate of 100 $\mathrm{MPa} / \mathrm{ps}$. The barostat ensures that the relative pressures in the three directions conform to the $\lambda$ vector for the target triaxial stress state. The simulation is terminated shortly after plasticity occurs, which is usually associated with GB dislocation nucleation.

\subsection{Analysis Methodology}

To analyze the conditions leading to GB dislocation nucleation over the range of triaxial stress states, each simulation must first be analyzed to determine the slip system on which dislocation nucleation occurs, and the resolved stresses associated with that nucleation event. As noted above, the work focuses on only one of the two GBs. Furthermore, because the analysis for 
nucleation from that GB into grains $A$ and $B$ are similar, most of the analysis focuses on nucleation into grain A, though some results for grain B are also included to show similarity.

For the Nickel GB analyzed here, partial dislocations with a trailing stacking fault are the primary dislocation nucleation event observed, so dislocation nucleation is analyzed in terms of the twelve $\{111\}\langle 11 \overline{2}\rangle$ partial dislocation slip systems. The stacking faults simplify the identification of the slip systems, because the atoms are easily identified by their CNA value. The slip plane is found by fitting a plane to the slipped atoms, while the slip direction is determined by examining the slip vector [31] of these same atoms.

In this work, the stresses that lead to nucleation are analyzed based on the local stress near the GB. The global stress is not used because it can differ significantly from the local stress where nucleation actually occurs. The stress exactly in the GB is also not used because the GB itself has a complicated stress state due to atomic disorder as well as the anisotropy caused by the differing orientations of the grain on either side. Instead, the local stress is determined by averaging the virial stress [32] of all atoms between 5 and $10 \AA ̊$ from the center of the GB as illustrated in Figure 3a. As will be seen in the results and discussion, this approach leads to reasonably good criteria for nucleation.

Once the local stress state and slip system have been identified, the resolved stresses acting on this slip system in the atoms between 5 and $10 \AA$ from the center of the GB are determined (Figure 3a). Figure $3 \mathrm{~b}$ plots the time evolution of the resolved shear, normal, and co-slip stress components for the slip system on which nucleation occurs. The drop in resolved shear stress corresponds to a dislocation nucleation event at the GB. Following the stress drop, the stress states fluctuate significantly. Some of this fluctuation is a natural result of slip propagation, while some results from attempts by the barostat to regulate the stress. Our examination focuses on the stress sampled just prior to the maximum value, therefore, these fluctuations are of no concern in our analysis.

The stresses that lead to nucleation are sampled $20 \mathrm{fs}$ prior to the maximum in the resolved shear stress on the nucleation slip system. This ensures that the measured stress precedes instabilities in the stress condition caused by the nucleation event. The sampling time for these 
conditions is identified as the red vertical line in Figure $3 b$, which occurs just prior to the maximum resolved shear stress, identified as the blue vertical line. When nucleation occurs on two different slip systems simultaneously, the first of the two maxima is chosen to identify the time of the dislocation nucleation event. Using this approach to extract the critical conditions surrounding nucleation, GB dislocation nucleation on the different slip systems can be compared across the 386 triaxial stress states.

\section{Results}

Representative GB dislocation nucleation responses for the 386 different triaxial stress states are presented in Figure 4, where the atoms are colored by their CNA value. Of the 386 different simulations analyzed for grain A, 254 exhibit dislocation nucleation on a single slip system, 118 exhibit dislocation on two different slip systems, and 14 exhibit plasticity of some sort that was not readily categorizable. The latter 14 cases are excluded from the remaining analysis.

Figure 4a shows an example case where each grain nucleates partial dislocations on a single slip system. Figure $4 b$ shows a case where two different slip systems exhibit dislocation nucleation. In this particular case, there are two partial dislocations that share a common slip plane, but which have different slip directions. The two partials combine to give the middle plane of atoms a net full dislocation. Figure $4 \mathrm{c}$ shows a combination of single slip system nucleation and dual slip system nucleation. In total, cases similar to Figures 4b and 4c account for 102 of the 118 cases that had some form of dual slip on a single slip plane. For these 102 multi slip-system events, only 7 resulted in nucleation events that were so indistinguishably close in time that the nucleation conditions must be considered for both slip systems. For the remaining 95 cases, one partial emerges first, followed by a second; this fine distinction is not apparent in the figures because Figure 4 demonstrates the dislocations well past their nucleation events. In these 95 cases, the nucleation conditions are only considered for the first slip system to exhibit nucleation. Finally, Figure $4 d$ shows a case where two different slip systems exhibit dislocation nucleation, but on different slip planes. These account for the final 16 cases, all of which exhibit simultaneous nucleation, and as such the nucleation conditions are considered for both slip systems. 
Over the full range of 386 triaxial stress states, GB dislocation nucleation was observed on only six of the twelve possible slip systems in each grain. The relationship between nucleated slip system and local stress state is represented schematically in Figure 5. Since the target triaxial stress state is given by the $\lambda$ vector, which resides on a unit sphere, the data is presented on stereographic maps for each grain. The left map is for simulations with $\sigma_{x} \geq 0$, that is, tensile $\sigma_{x}$. The right map is for simulations with $\sigma_{x}<0$, that is, compressive $\sigma_{x}$. It is smaller than the left map because it omits the $\sigma_{x}=0$ simulations that are already present on the perimeter of the left map. The colors on the maps represent the different slip systems on which GB dislocation nucleation occurs, as given by the legend for each grain. Simulations with simultaneous nucleation on two slip systems are thatched with both colors. The black tiles represent simulations that are dropped from the analysis as noted above.

A brief examination of Figure 5 reveals distinctive regions of triaxial stress that associate with specific slip systems. These regions converge to points of hydrostatic tension and compression $\left(\lambda= \pm\left[\frac{1}{\sqrt{3}}, \frac{1}{\sqrt{3}}, \frac{1}{\sqrt{3}}\right]\right)$. Furthermore, a brief comparison of the maps for grain A and grain $\mathrm{B}$ reveals a strong match between areas nucleating on different slip systems in the two grains, presumably due to the alignment of the two crystals and the symmetry of the GB. It is also worth noting that simulations that are dropped from the analysis (black tiles) occur primarily near the hydrostatic tension and compression regions, though some others occur near regions that transition from one slip system to another.

Figure 6 shows the magnitude of the resolved shear stress on the nucleated slip system just prior to nucleation for Grain A. Regions of similar resolved shear stress states correlate well with the regions of individual slip system activity in Figure 5. However, it is clear that different slip systems respond to different levels of resolved shear stress; furthermore, even within the region relating to a single nucleation slip system there is a gradient of resolved shear strengths, which indicates possible non-Schmid effects.

Also indicative of non-Schmid effects is the fact that for any given simulation, the system that exhibits nucleation will not necessarily have the highest magnitude of resolved shear stress, when compared with the resolved shear stress on the other 12 slip systems. This is 
demonstrated in Figure 7 where the resolved shear stress for each partial slip system is plotted as a function of time for the $\lambda=[-0.153,-0.699,0.699]$ simulation. GB dislocation nucleation occurs on the $(11 \overline{1})[\overline{2} 1 \overline{1}]$ slip system and is shown as a heavy red line. A heavy dotted red line shows the negative of the resolved shear for comparison with slip systems that have negative resolved shear stress. As can be seen, the red lines (solid and dashed) are lower in magnitude (absolute value) than those for three other slip systems that do not nucleate a dislocation.

To demonstrate the influence of other resolved stresses known to be of importance $[5,11]$, we examine the resolved normal, $\sigma_{r n s}$, and co-slip, $\tau_{r c o}$, stresses in addition to the resolved shear stress, $\tau_{r s s}$. Figure 8a plots the sampled stress values of $\tau_{r s s}, \sigma_{r n s}$ and $\tau_{r c o}$ resolved on to the (111) [2 111$]$ slip system for each of the 386 simulations in grain A, regardless of whether nucleation occurred on the (111)[211] slip system. The dropped simulations (black tiles in Figure 5) are not included. In other words, each point corresponds to the resolved stresses on the (111)[211] slip system at the sampling time when nucleation was detected in that simulation even if nucleation occurred on another system. Stress levels that nucleate dislocations on the (111)[2 111$]$ slip system are marked as closed circles, while those that nucleate on another slip system are marked as open circles, allowing the immediate comparison of stress levels that are associated with nucleation and those that were not, for a single slip system. This process is repeated for the other 5 nucleating slip systems in Figure 8b8f. If simultaneous nucleation is observed on two slip systems, both slip systems are indicated and the point is included as a closed circle on the graphs for both slip systems.

In examining the figures, it can be observed that the closed circles are generally associated with high levels of $\tau_{r s s}$, and tend to cluster along a plane in the 3D space. To demonstrate and quantify the normal and co-slip stress dependencies, a plane is fit to the nucleating (closed circle) points using the form

$$
1=\frac{\tau_{r s s}}{\tau_{c r s s}}+\frac{\sigma_{r n s}}{\sigma_{c r n s}}+\frac{\tau_{r c o}}{\tau_{c r c o}}
$$

The parameters $\tau_{c r s s}, \sigma_{c r n s}$, and $\tau_{c r c o}$ are fitting parameters and correspond to the intercepts of the plane on the $\tau_{r s s}, \sigma_{r n s}$ and $\tau_{r c o}$ axes, respectively. 
The planar fitting parameters for nucleation on the six slip systems in Figure 8 are shown in Table 1. The fitting parameters for nucleation in grain B are shown in Table 2. These tables also include the $R^{2}$ value for the planar fit and the number of simulations, $N$, exhibiting nucleation on the slip system. Each grain has its own coordinate system so the slip systems cannot be compared across the two tables.

\section{Discussion}

The exploration of a large set of triaxial stress states provides surprising insight into dislocation nucleation at GBs. Dislocation nucleation criteria can be formulated, from which nucleation surfaces for GB dislocation nucleation can be created. These and other implications resulting from the present work are discussed in the following sections.

\subsection{Nucleation Criteria}

The planar fits to the resolved shear, normal, and co-slip stresses that correspond to dislocation nucleation provide unique insight into the conditions under which nucleation occurs. By definition of Eq. 2, the values $\tau_{c r s s}, \sigma_{c r n s}$, and $\tau_{c r c o}$ in Tables 1 and 2 define the stress components required to achieve nucleation on the respective slip system. It is noted that some of the $R^{2}$ values are low; despite the visually appealing fit, the planar model does not always appear to be the best description for the distribution of the points. Residual analysis indicates that non-linear models might improve the prediction of resolved stresses required for nucleation; this is left for future research. Nonetheless, the results are appealing and there appear to be a small number of critical values arising from the linear analysis that can describe the local stress dependence for nucleation on a given slip system.

Since resolved shear stress is known to be the main factor in dislocation glide [33], it is

unsurprising that the $\tau_{\text {crss }}$ fitting parameters are substantially smaller than the $\sigma_{c r n s}$ and $\tau_{c r c s}$ values; initiating GB dislocation nucleation without any resolved shear stress is difficult. The critical $\tau_{c r s s}, \sigma_{c r n s}$, and $\tau_{c r c o}$ values that result from the planar fit essentially define a criterion for GB dislocation nucleation on the various slip systems. In other words, the equality in Eq. 2 can be turned into the inequality 


$$
1 \leq \frac{\tau_{r s s}}{\tau_{c r s s}}+\frac{\sigma_{r n s}}{\sigma_{c r n s}}+\frac{\tau_{r c o}}{\tau_{c r c o}}
$$

such that stress states below the plane (that is, stress states for which Eq. 3 is false) are not expected to nucleate dislocations on that slip system, and stress states above the plane should nucleate on that slip system. By applying this criterion to each of the six active systems, and identifying the system with the lowest stress for each triaxial state, predictions of the nucleating slip system are plotted in Figure 9a. The observed nucleation slip systems for grain A are plotted in Figure 9b for comparison. Despite the rudimentary nature of the linear approximation for the nucleation criteria, the match with test results is remarkable; 362 of the 372 cases $(97.3 \%)$ match (in the case of multiple slip nucleation, if at least one of the two is accurate it is considered a match). However, there are points of difference and the model was not always accurate despite being trained on the data to which it is compared. Examination of other resolved stress components, such as a normal stress in the direction of the Burgers vector, suggests that these could also be critical to nucleation in some cases. Nonetheless, no other single model, similar to Eq. 3, could be applied to all active slip systems and provide consistent predictions. Instead, inclusion of additional components leads to different criteria for each slip system. This potential for change in relative importance of different stress components was suggested by Spearot et al. who found that dislocation nucleation on the $\langle 100\rangle$ GBs only had normal stress dependence while the $\langle 110\rangle$ GBs had only shear stress dependence; neither had co-slip shear stress dependence despite the hypothesized dependence upon all three resolved stress components [5]. Additional work is needed in this area to determine if one criteria can be consistently used, even if it is not perfect, or if each GB and slip system is so unique that each must have its own nucleation criterion.

As a point of comparison to other nucleation models, we consider correlations between nucleation slip system and the related Schmid factor, which has been proposed as a useful metric in determining the slip system for GB dislocation nucleation [18,34]. If the slip system for nucleation is predicted by the system with the maximum Schmid factor, using the stresses at the point of nucleation, one obtains the prediction given in Figure 9c. Here it can be seen that only 142 of the 372 cases (38.2\%) match. As noted by others, nucleation frequently occurs on a slip system other than that predicted by a Schmid factor analysis $[6,17,35]$. Furthermore, the 
Schmid prediction suggests nucleation should occur on slip systems that are never observed to be active in the simulations.

To show the importance of the non-Schmid components and provide physical insight, the planar Eq. (2) can be rearranged. With simple algebraic manipulation, Eq. (2) can be changed into the following functional form

$$
\tau_{r S s}=\tau_{c r s s}+\frac{\partial \tau_{r s s}}{\partial \sigma_{r n s}} \sigma_{r n s}+\frac{\partial \tau_{r s s}}{\partial \tau_{r c o}} \tau_{r c o} .
$$

In Eq. (4), $\frac{\partial \tau_{r s s}}{\partial \sigma_{r n s}}=-\frac{\tau_{c r s s}}{\sigma_{c r n s}}$ and $\frac{\partial \tau_{r s s}}{\partial \tau_{r c o}}=-\frac{\tau_{c r s s}}{\tau_{c r c o}}$ are substituted to denote the dependence of the resolved shear stress at nucleation on the resolved normal and co-slip stress. In other words, the partial differentials capture the slope of the critical nucleation plane in these two independent directions, though it is noted that these partial differentials, or slopes, are constants. This change of variables mimics the Mohr-Coulomb yield criterion, where the critical shear stress for yield is known to have a normal stress dependence. The value of $\frac{\partial \tau_{r S s}}{\partial \sigma_{r n s}}$ is expected to be negative (meaning larger tensile resolved normal stresses lower the needed resolved shear stress for nucleation) because compressive normal stresses increase internal friction, making it harder for the atoms on a slip system to glide over each other $[5,11,36]$. The functional fitting parameters $\frac{\partial \tau_{r s s}}{\partial \sigma_{r n s}}$ and $\frac{\partial \tau_{r s s}}{\partial \tau_{r c o}}$ for each slip system and grain are shown in Tables 1 and 2. As expected, $\frac{\partial \tau_{r s s}}{\partial \sigma_{r n s}}$ is negative for each slip system. In contrast, $\frac{\partial \tau_{r s s}}{\partial \tau_{r c o}}$ can be both positive and negative depending upon the slip system since this indicates directionality of the shear stress.

For the present work on a single GB, the fact that each slip system has a different nucleation criterion begs an important question: does the change in criteria result from a geometrical difference between slip systems, the grain boundary, and the alignment of the stress, or does it result from a difference in atomic structure of the GB for each slip system? A geometrical difference would result from the fact that different slip planes and directions have different angles relative to the GB, so slip planes that are perpendicular might favor nucleation while planes that are nearly parallel to the GB plane might inhibit nucleation. A structural difference 
of the atoms in the GB could result in some slip systems being well aligned with features of the GB structure to favor dislocation nucleation while other slip systems could be poorly aligned with the same features of the GB structure and therefore inhibit dislocation nucleation.

In the work of Spearot et al. a variety of similar GBs under a single stress state were examined [5]. The same primary slip system was examined over variations of tilt angle for $\langle 100\rangle$ and $\langle 110\rangle$ axes. Based on this evidence, one could reasonably assume that the GB structure is similar between these GBs, thereby attributing the variation to the geometry.

In the present work, an attempt was made to attribute the variation to geometry. This effort involved examining possible relationships between the $\tau_{c r s s}, \frac{\partial \tau_{r s s}}{\partial \sigma_{r n s}}$, and $\frac{\partial \tau_{r s s}}{\partial \tau_{r c o}}$ coefficients and the orientation of each slip system's slip plane and slip direction with respect to the GB plane. No patterns were observed. It could be that with only 12 data points between Tables 1 and 2, there may be geometrical relationships that simply require additional data to extract, but which are not immediately obvious. However, since all 12 data points in Tables 1 and 2 have unique criteria and display no immediate geometrical relationship with the $G B$, this suggests to the authors that the atomic structure is playing an important and unique role for each slip system where it emerges from the GB. This argument is supported by the fact that some GBs can shear-couple because the GB structure has dislocation content that facilitates shear while other GBs do not provide this amenable dislocation content, and cannot shear couple as a result $[37,38]$.

If the atomic structure does play a critical role in the variation of GB dislocation nucleation criteria, different GBs will have different nucleation criteria because the structure is different. A comparison of the present work with that of Spearot et al. would seem to support this argument because the criteria are different for two different GBs. However, it is more complicated than this because the present work uses a Ni EAM potential [26], while Spearot et al. used a Cu EAM potential [39]. The two potentials have different stacking fault energies, among other properties, leading to partial vs full GB dislocation nucleation. The different potentials can lead to different GB structures for the same GB misorientation and boundary plane orientation. The different structures would then have different criteria for partial vs full 
dislocation nucleation, again supporting the importance of the role of structure, which is defined by the atomic potentials.

Together, the present work and the work of Spearot et al. indicate that both geometry and atomic structure are important factors for the GB dislocation nucleation criteria. If true, there are likely to be some criteria that vary only with geometry when GBs have similar structure, as in the case of Spearot et al. But different GB structures, and even different slip systems for a given $\mathrm{GB}$, are likely to have unique criteria because the atomic structure can be different, as in the case of the present work.

It is also important to note that the nucleation criteria presented in this work are derived from the GB studied in its present configuration. It is not clear how these criteria will change when more complex structures are considered, such as non-equilibrium interface structures [18], GB ledges [40,41], triple junctions [16], or nanoporous GBs [14]. It is possible that non-equilibrium GBs will only slightly modify the nucleation criteria, while GB ledges will significantly reduce the required stresses for some slip systems. These structural variations might also lead to different phenomena, as indicated by Tucker, et al., who showed that non-equilibrium content added to a GB can lead to GB migration instead of GB dislocation nucleation [7]. Because the nucleation criteria appear to be influenced by the GB structure, each structural feature (ledges, etc.) will need to be studied in a large number of GBs to understand how they influence GB dislocation nucleation.

\subsection{Grain Boundary Dislocation Nucleation Surfaces}

By combining GB dislocation nucleation criteria for all 6 slip systems that are activated, a single nucleation criterion can be formed for the GB. A nucleation surface is built for the GB using principal stresses oriented in the $\hat{x}, \hat{y}$, and $\hat{z}$ directions by finding the first slip system nucleation criterion that matches a principal stress state moving outward from the origin. The nucleation surface is shown in Figures 10a and 10b for grain A. Each facet corresponds to GB dislocation nucleation on a different slip system. The facets are colored using the same color coding as in Figure 5a. A dashed line of hydrostatic stress is included for reference. A biaxial nucleation surface slice for $\sigma_{x}=0$ is shown in Figure 10c. 
The GB dislocation nucleation surface is reminiscent of a Mohr-Coulomb yield surface [42]. The nucleation surface is well aligned with the line of hydrostatic stress suggesting nucleation is largely a function of deviatoric stress, though not independent of other factors. Not surprisingly, the GB is particularly strong under triaxial compression. In Mohr-Coulomb, each facet of the yield surface arises from the two principal stresses that produce the maximal shear; in the GB dislocation nucleation surface each facet corresponds to GB dislocation nucleation on a different slip system.

While the nucleation surface is instructive, it is important to note that it is not a failure envelope in the traditional sense. This nucleation surface has a specific orientation, in that the principal stresses must be in the same reference frame as the GB. As such, one cannot use the plot to consider stress states where the shear stresses are non-zero; but this is a limitation of the plot, not the nucleation criterion used to construct the plot. The nucleation criteria for each slip system are in the reference frame of the slip system; therefore, any arbitrary stress state can be resolved into this slip system and be considered. It is for this reason that only five of the six nucleation criteria for grain A show up as faces on the nucleation surface; in the principal stress space one of the nucleation criteria is always activated before the unrepresented criterion. One nucleation criterion masks the other.

The nucleation surface does not account for GB plasticity mechanisms other than GB dislocation nucleation; however, if criteria to activate other plasticity mechanisms (such as GB sliding or GB migration) can be formulated as a function of stress state, they could be combined with our nucleation surface to form a more comprehensive plasticity envelope.

\section{Conclusion}

The present work provides insight into the criteria associated with dislocation nucleation at grain boundaries. The criteria are investigated using molecular dynamics simulations of a highangle $\Sigma 21 \mathrm{~b}$ symmetric twist grain boundary under a range of triaxial stresses. Local stresses near the GB are examined for their influence on dislocation nucleation from the grain boundary on the various slip systems. Based on this work the following conclusions can be drawn: 
- The use of different triaxial stress states leads to a rich response of GB dislocation nucleation on different slip systems and at different stresses, enabling criteria to be determined for the influence of stress on the nucleation event of specific slip systems.

- The resolved shear stress for dislocation nucleation at grain boundaries has a strong dependence on the resolved normal and resolved co-slip stresses. This supports and contrasts previous observations where resolved normal dependence was observed and resolved co-slip was investigated but not observed $[5,11]$.

- Each slip system at a GB appears to have its own nucleation criteria, and a unique stress dependence. The unique criteria appear to be based on GB structure, rather than on the alignment of slip systems with the GB plane.

- A theoretical nucleation surface can be constructed from the combination of the various GB dislocation nucleation criteria. The nucleation surface is reminiscent of a Mohr-Coulomb yield surface, though each facet of the nucleation surface is due to nucleation on a different slip system.

While the current work does indicate that much is yet to be learned about dislocation nucleation at GBs, the portrayed approach provides a new methodology to thoroughly investigate the phenomenon, and the resultant nucleation criteria provide a mechanism to pass the work to higher length scales, such as finite element crystal plasticity models.

\section{Acknowledgments}

This work was supported by the U.S. Department of Energy, Office of Science, Basic Energy Sciences under Award \# DE-SC0012587. The authors also wish to thank the Fulton Supercomputing Lab staff at Brigham Young University for their assistance with the computational work.

\section{References}

[1] G.Z. Voyiadjis, M. Yaghoobi, Role of grain boundary on the sources of size effects, Comput. Mater. Sci. 117 (2016) 315-329. doi:10.1016/j.commatsci.2016.01.025.

[2] H. Van Swygenhoven, Grain Boundaries and Dislocations, Science (80-. ). 296 (2002) 66- 
67. doi:110.1126/science.1071040.

[3] A.C. Lund, C.A. Schuh, Strength asymmetry in nanocrystalline metals under multiaxial loading, Acta Mater. 53 (2005) 3193-3205. doi:10.1016/j.actamat.2005.03.023.

[4] D. Wolf, V. Yamakov, S.R. Phillpot, A. Mukherjee, H. Gleiter, Deformation of nanocrystalline materials by molecular-dynamics simulation: relationship to experiments?, Acta Mater. 53 (2005) 1-40. doi:10.1016/j.actamat.2004.08.045.

[5] D. Spearot, M. Tschopp, K. Jacob, D. McDowell, Tensile strength of $\langle 100\rangle$ and $\langle 110\rangle$ tilt bicrystal copper interfaces, Acta Mater. 55 (2007) 705-714.

doi:10.1016/j.actamat.2006.08.060.

[6] I.J. Beyerlein, J. Wang, R. Zhang, Mapping dislocation nucleation behavior from bimetal interfaces, Acta Mater. 61 (2013) 7488-7499. doi:10.1016/j.actamat.2013.08.061.

[7] G.J. Tucker, D.L. McDowell, Non-equilibrium grain boundary structure and inelastic deformation using atomistic simulations, Int. J. Plast. 27 (2011) 841-857. doi:10.1016/j.ijplas.2010.09.011.

[8] M.A. Tschopp, D.E. Spearot, D.L. McDowell, Atomistic simulations of homogeneous dislocation nucleation in single crystal copper, Model. Simul. Mater. Sci. Eng. (2007) 693709. doi:10.1063/1.2715137.

[9] M.A. Tschopp, D.L. McDowell, Influence of single crystal orientation on homogeneous dislocation nucleation under uniaxial loading, J. Mech. Phys. Solids. 56 (2008) 1806-1830. doi:10.1016/j.jmps.2007.11.012.

[10] S. Ogata, J. Li, S. Yip, Ideal pure shear strength of aluminum and copper., Science. 298 (2002) 807-811. doi:10.1126/science.1076652.

[11] K. Kinoshita, T. Shimokawa, T. Kinari, Grain Boundary Structure Dependence of Extrinsic Grain Boundary Dislocation Emission Phenomena: A Molecular Dynamics Study, Mater. Trans. 53 (2012) 147-155. doi:10.2320/matertrans.MD201124.

[12] M.D. Sangid, T. Ezaz, H. Sehitoglu, I.M. Robertson, Energy of slip transmission and 
nucleation at grain boundaries, Acta Mater. 59 (2011) 283-296.

doi:10.1016/j.actamat.2010.09.032.

[13] L. Capolungo, D.E. Spearot, M. Cherkaoui, D.L. McDowell, J. Qu, K.I. Jacob, Dislocation nucleation from bicrystal interfaces and grain boundary ledges: Relationship to nanocrystalline deformation, J. Mech. Phys. Solids. 55 (2007) 2300-2327. doi:10.1016/j.jmps.2007.04.001.

[14] D.E. Spearot, Evolution of the E structural unit during uniaxial and constrained tensile deformation, Mech. Res. Commun. 35 (2008) 81-88. doi:10.1016/j.mechrescom.2007.09.002.

[15] Y.-J. Wang, A. Ishii, S. Ogata, Transition of creep mechanism in nanocrystalline metals, Phys. Rev. B. 84 (2011) 224102. doi:10.1103/PhysRevB.84.224102.

[16] Z.X. Wu, Y.W. Zhang, M.H. Jhon, D.J. Srolovitz, Anatomy of nanomaterial deformation: Grain boundary sliding, plasticity and cavitation in nanocrystalline Ni, Acta Mater. 61 (2013) 5807-5820. doi:10.1016/j.actamat.2013.06.026.

[17] R.F. Zhang, J. Wang, I.J. Beyerlein, T.C. Germann, Dislocation nucleation mechanisms from fcc/bcc incoherent interfaces, Scr. Mater. 65 (2011) 1022-1025. doi:10.1016/j.scriptamat.2011.09.008.

[18] N.J. Burbery, R. Das, W.G. Ferguson, Modelling with variable atomic structure: Dislocation nucleation from symmetric tilt grain boundaries in aluminium, Comput. Mater. Sci. 101 (2015) 16-28. doi:10.1016/j.commatsci.2015.01.010.

[19] D. Faken, H. Jónsson, Systematic analysis of local atomic structure combined with 3D computer graphics, Comput. Mater. Sci. 2 (1994) 279-286. doi:10.1016/09270256(94)90109-0.

[20] D.L. Olmsted, S.M. Foiles, E.A. Holm, Survey of computed grain boundary properties in face-centered cubic metals: I. Grain boundary energy, Acta Mater. 57 (2009) 3694-3703. doi:10.1016/j.actamat.2009.04.007. 
[21] D.L. Olmsted, E.A. Holm, S.M. Foiles, Survey of computed grain boundary properties in face-centered cubic metals - II: Grain boundary mobility, Acta Mater. 57 (2009) 37043713. doi:10.1016/j.actamat.2009.04.015.

[22] S. Plimpton, Fast Parallel Algorithms for Short-Range Molecular Dynamics, J. Comput. Phys. 117 (1995) 1-19. doi:http://dx.doi.org/10.1006/jcph.1995.1039.

[23] W.M. Brown, P. Wang, S.J. Plimpton, A.N. Tharrington, Implementing molecular dynamics on hybrid high performance computers - short range forces, Comput. Phys. Commun. 182 (2011) 898-911. doi:10.1016/j.cpc.2010.12.021.

[24] W.M. Brown, A. Kohlmeyer, S.J. Plimpton, A.N. Tharrington, Implementing molecular dynamics on hybrid high performance computers - Particle-particle particle-mesh, Comput. Phys. Commun. 183 (2012) 449-459. doi:10.1016/j.cpc.2011.10.012.

[25] W.M. Brown, M. Yamada, Implementing molecular dynamics on hybrid high performance computers-Three-body potentials, Comput. Phys. Commun. 184 (2013) 2785-2793. doi:10.1016/j.cpc.2013.08.002.

[26] S. Foiles, J. Hoyt, Computation of grain boundary stiffness and mobility from boundary fluctuations, Acta Mater. 54 (2006) 3351-3357. doi:10.1016/j.actamat.2006.03.037.

[27] A. Stukowski, Visualization and analysis of atomistic simulation data with OVITO-the Open Visualization Tool, Model. Simul. Mater. Sci. Eng. 18 (2009) 015012. doi:10.1088/0965-0393/18/1/015012.

[28] S. Nosé, A unified formulation of the constant temperature molecular dynamics methods, J. Chem. Phys. 81 (1984) 511. doi:10.1063/1.447334.

[29] W.G. Hoover, Canonical dynamics: Equilibrium phase-space distributions, Phys. Rev. A. 31 (1985) 1695-1697. doi:10.1103/PhysRevA.31.1695.

[30] A. Semechko, Suite of functions to perform uniform sampling of a sphere, (2015). https://www.mathworks.com/matlabcentral/fileexchange/37004-suite-of-functions-toperform-uniform-sampling-of-a-sphere (accessed April 19, 2016). 
[31] J. a. Zimmerman, C.L. Kelchner, P. a Klein, J.C. Hamilton, S.M. Foiles, Surface step effects on nanoindentation., Phys. Rev. Lett. 87 (2001). doi:10.1103/PhysRevLett.87.165507.

[32] A.P. Thompson, S.J. Plimpton, W. Mattson, General formulation of pressure and stress tensor for arbitrary many-body interaction potentials under periodic boundary conditions., J. Chem. Phys. 131 (2009) 154107. doi:10.1063/1.3245303.

[33] E. Schmid, W. Boas, Kristallplastizität, Springer - Verlag Berlin Heidelberg, 1935. doi:10.1007/978-3-662-34532-0.

[34] D.E. Spearot, K.I. Jacob, D.L. McDowell, Nucleation of dislocations from [001] bicrystal interfaces in aluminum, Acta Mater. 53 (2005) 3579-3589.

doi:10.1016/j.actamat.2005.04.012.

[35] D.E. Spearot, K. Jacob, D. McDowell, Dislocation nucleation from bicrystal interfaces with dissociated structure, Int. J. Plast. 23 (2007) 143-160. doi:10.1016/j.ijplas.2006.03.008.

[36] I. Salehinia, D.F. Bahr, Crystal orientation effect on dislocation nucleation and multiplication in FCC single crystal under uniaxial loading, Int. J. Plast. 52 (2014) 133-146. doi:10.1016/j.ijplas.2013.04.010.

[37] J.W. Cahn, Y. Mishin, A. Suzuki, Duality of dislocation content of grain boundaries, Philos. Mag. 86 (2006) 3965-3980. doi:10.1080/14786430500536909.

[38] E.R. Homer, S.M. Foiles, E.A. Holm, D.L. Olmsted, Phenomenology of shear-coupled grain boundary motion in symmetric tilt and general grain boundaries, Acta Mater. 61 (2013) 1048-1060. doi:10.1016/j.actamat.2012.10.005.

[39] Y. Mishin, M.J. Mehl, D.A. Papaconstantopoulos, A.F. Voter, J.D. Kress, Structural stability and lattice defects in copper: Ab initio, tight-binding, and embedded-atom calculations, Phys. Rev. B. 63 (2001) 224106. doi:10.1103/PhysRevB.63.224106.

[40] M.G. McPhie, S. Berbenni, M. Cherkaoui, Activation energy for nucleation of partial dislocation from grain boundaries, Comput. Mater. Sci. 62 (2012) 169-174. doi:10.1016/j.commatsci.2012.05.039. 
[41] A. Hunter, I.J. Beyerlein, Stacking fault emission from grain boundaries: Material dependencies and grain size effects, Mater. Sci. Eng. A. 600 (2014) 200-210. doi:10.1016/j.msea.2014.02.030.

[42] M. Yu, Advances in strength theories for materials under complex stress state in the 20th Century, Appl. Mech. Rev. 55 (2002) 169-218. http://dx.doi.org/10.1115/1.1472455. 


\section{Tables}

Table 1. List of slip system specific coefficients, $\tau_{\text {crss }}, \sigma_{\text {crns }}$, and $\tau_{\text {crco }}$, for GB dislocation nucleation into grain A as defined by Eqs. 2 and 3. The number of datapoints, $N$, for the planar fit and the goodness of fit, $R^{2}$, are also included. The normal and co-slip stress dependence of the criteria are given as $\frac{\partial \tau_{r s s}}{\partial \sigma_{r n s}}$ and $\frac{\partial \tau_{r s S}}{\partial \tau_{r c o}}$ as defined by Eq. 4 .

\begin{tabular}{|c|c|c|c|c|c|c|c|}
\hline Slip System & $\tau_{\text {crss }}$ & $\sigma_{\text {crns }}$ & $\tau_{\text {crco }}$ & $\frac{\partial \tau_{r s s}}{\partial \sigma_{r n s}}$ & $\frac{\partial \tau_{r s s}}{\partial \tau_{r c o}}$ & $N$ & $R^{2}$ \\
\hline$(11 \overline{1})[\overline{2} 11]$ & $4.38 \mathrm{GPa}$ & $44.3 \mathrm{GPa}$ & $7.70 \mathrm{GPa}$ & -0.099 & -0.569 & 12 & 0.986 \\
\hline$(1 \overline{1} 1)[1 \overline{1} 1]$ & $3.42 \mathrm{GPa}$ & $35.6 \mathrm{GPa}$ & $-16.3 \mathrm{GPa}$ & -0.096 & 0.210 & 17 & 0.970 \\
\hline$(1 \overline{1} 1)[1 \overline{1}]]$ & $5.68 \mathrm{GPa}$ & $41.5 \mathrm{GPa}$ & $18.3 \mathrm{GPa}$ & -0.137 & -0.311 & 44 & 0.679 \\
\hline$(11 \overline{1})[\overline{2} 1 \overline{1}]$ & $3.38 \mathrm{GPa}$ & $62.7 \mathrm{GPa}$ & $-42.3 \mathrm{GPa}$ & -0.054 & 0.080 & 106 & 0.616 \\
\hline$(11 \overline{1})[1 \overline{2} \overline{1}]$ & $3.88 \mathrm{GPa}$ & $49.7 \mathrm{GPa}$ & $8.19 \mathrm{GPa}$ & -0.078 & -0.474 & 62 & 0.875 \\
\hline$(11 \overline{1})[112]$ & $1.58 \mathrm{GPa}$ & $117 \mathrm{GPa}$ & $6.79 \mathrm{GPa}$ & -0.014 & -0.233 & 154 & 0.654 \\
\hline
\end{tabular}

Table 2. List of slip system specific coefficients, $\tau_{c r s s}, \sigma_{c r n s}$, and $\tau_{c r c o}$, for GB dislocation nucleation into grain B as defined by Eqs. 2 and 3. The number of datapoints, $N$, for the planar fit and the goodness of fit, $R^{2}$, are also included. The normal and co-slip stress dependence of the criteria are given as $\frac{\partial \tau_{r s s}}{\partial \sigma_{r n s}}$ and $\frac{\partial \tau_{r S S}}{\partial \tau_{r c o}}$ as defined by Eq. 4 .

\begin{tabular}{|c|c|c|c|c|c|c|c|}
\hline Slip System & $\tau_{\text {crss }}$ & $\sigma_{\text {crns }}$ & $\tau_{\text {crco }}$ & $\frac{\partial \tau_{r s s}}{\partial \sigma_{r n s}}$ & $\frac{\partial \tau_{r s s}}{\partial \tau_{r c o}}$ & $N$ & $R^{2}$ \\
\hline$(111)[\overline{2} 11]$ & $4.57 \mathrm{GPa}$ & $44.8 \mathrm{GPa}$ & $-9.82 \mathrm{GPa}$ & -0.102 & 0.466 & 17 & 0.981 \\
\hline$(1 \overline{1} 1)[\overline{2} \overline{1} 1]$ & $3.17 \mathrm{GPa}$ & $50.5 \mathrm{GPa}$ & $209 \mathrm{GPa}$ & -0.063 & -0.015 & 90 & 0.736 \\
\hline$(1 \overline{1} 1)[121]$ & $1.70 \mathrm{GPa}$ & $132 \mathrm{GPa}$ & $24.5 \mathrm{GPa}$ & -0.013 & -0.070 & 153 & 0.298 \\
\hline$(1 \overline{1} 1)[1 \overline{1} \overline{2}]$ & $3.07 \mathrm{GPa}$ & $76.2 \mathrm{GPa}$ & $-11.1 \mathrm{GPa}$ & -0.040 & 0.278 & 74 & 0.482 \\
\hline$(11 \overline{1})[1 \overline{21}]$ & $5.93 \mathrm{GPa}$ & $42.1 \mathrm{GPa}$ & $-37.9 \mathrm{GPa}$ & -0.141 & 0.156 & 44 & 0.896 \\
\hline$(11 \overline{1})[112]$ & $2.86 \mathrm{GPa}$ & $35.5 \mathrm{GPa}$ & $11.7 \mathrm{GPa}$ & -0.081 & -0.245 & 20 & 0.931 \\
\hline
\end{tabular}




\section{Figures}

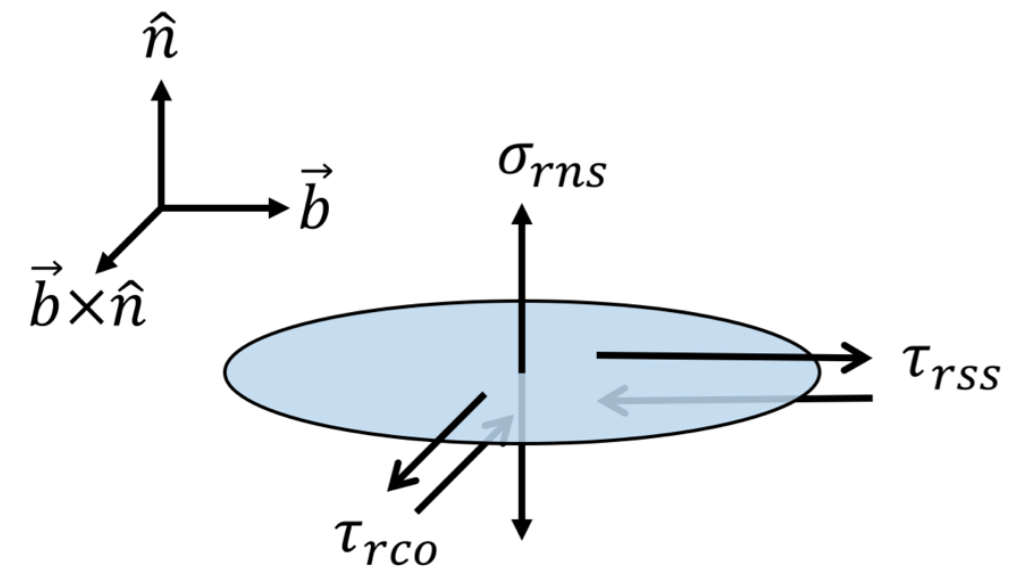

Figure 1. Resolved shear stress $\left(\tau_{\mathrm{rss}}\right)$, resolved normal stress $\left(\sigma_{\mathrm{rns}}\right)$ and resolved co-slip stress ( $\tau_{\text {rco }}$ ) shown schematically on a slip plane with normal $\hat{n}$, and $\tau_{\text {rss }}$ in the direction of the Burger's vector $\overrightarrow{\mathrm{b}}$. 


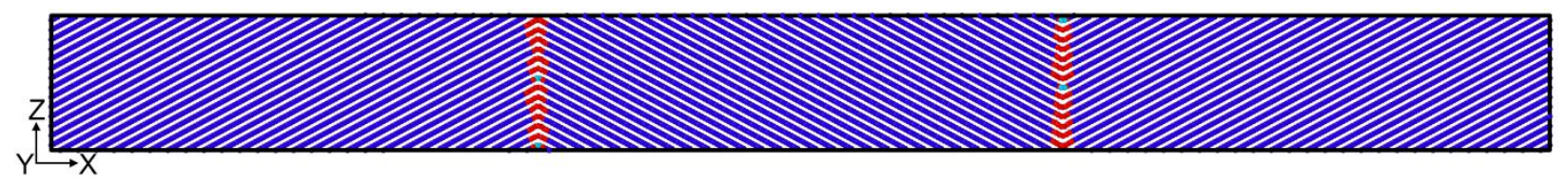

Figure 2. The $\Sigma 21 \mathrm{~b}$ [211] $44.415^{\circ}$ symmetric twist Nickel GBs used in this work. Atoms are colored by their common neighbor analysis (CNA) value (FCC = blue, HCP = turquoise, Undefined $=$ red). 
a)

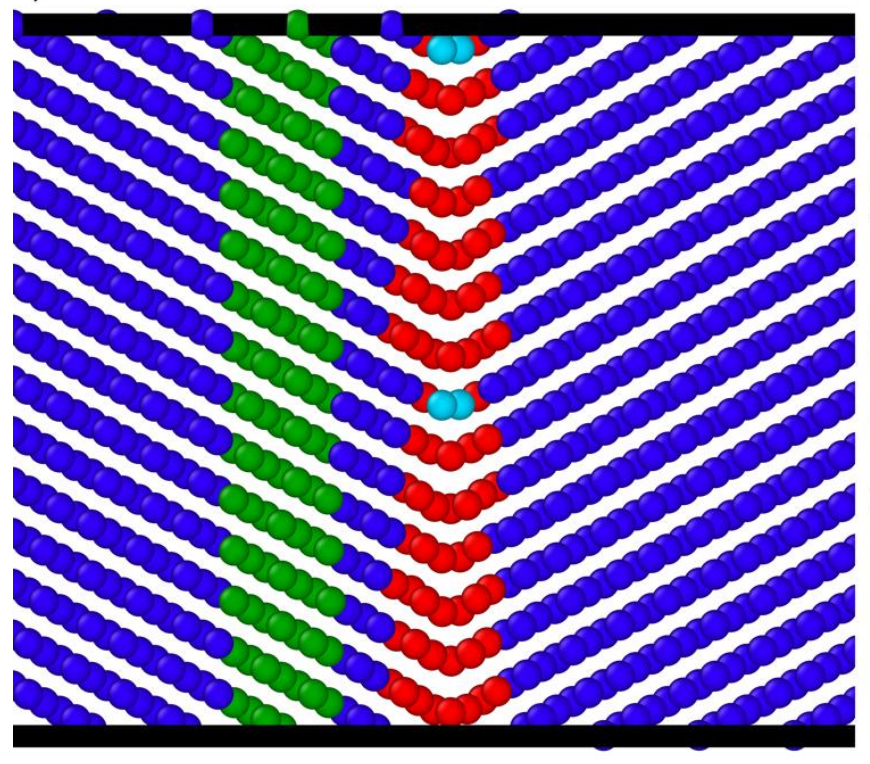

b)

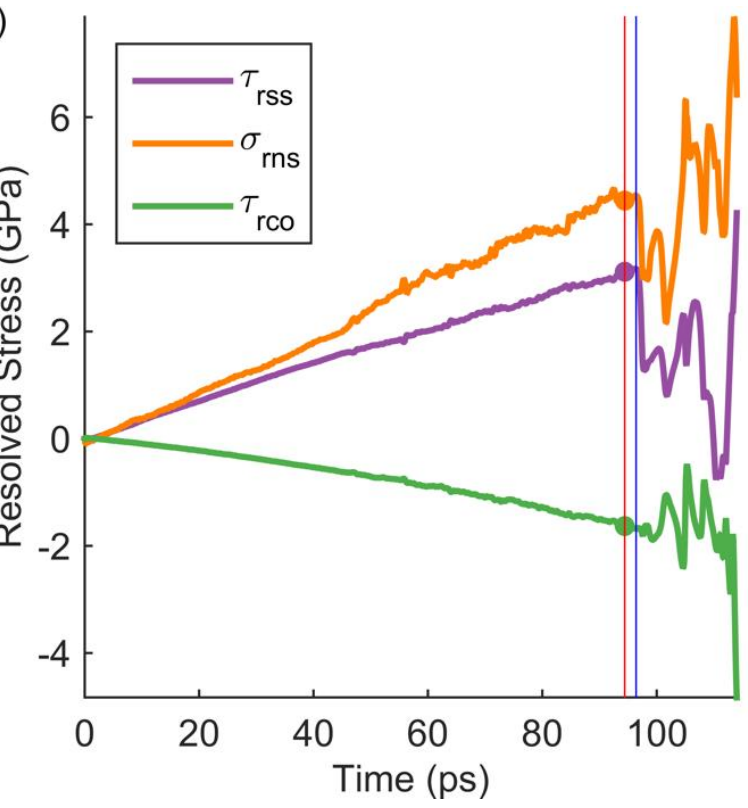

Figure 3. (a) Schematic of the atoms used to calculate the local stress state. The GB is marked by the atoms shown in red and turquoise, while the green atoms, between 5 and $10 \AA$ from the center of the GB, are used for the local stress calculations. The local stress is taken as the average of the green atoms' virial stress. (b) Evolution of the resolved shear, normal and co-slip stresses on the $(11 \overline{1})[\overline{2} 1 \overline{1}]$ slip system for the $\lambda=[-0.153,-0.699,0.699]$ simulation. The vertical blue line shows when $\tau_{r s s}$ is a maximum. The sudden drop in $\tau_{\text {rss }}$ correlates with the GB dislocation nucleation event on the (111) $[\overline{2} 1 \overline{1}]$ slip system. Stresses are sampled 20 ps prior to the maximum (vertical red line). 

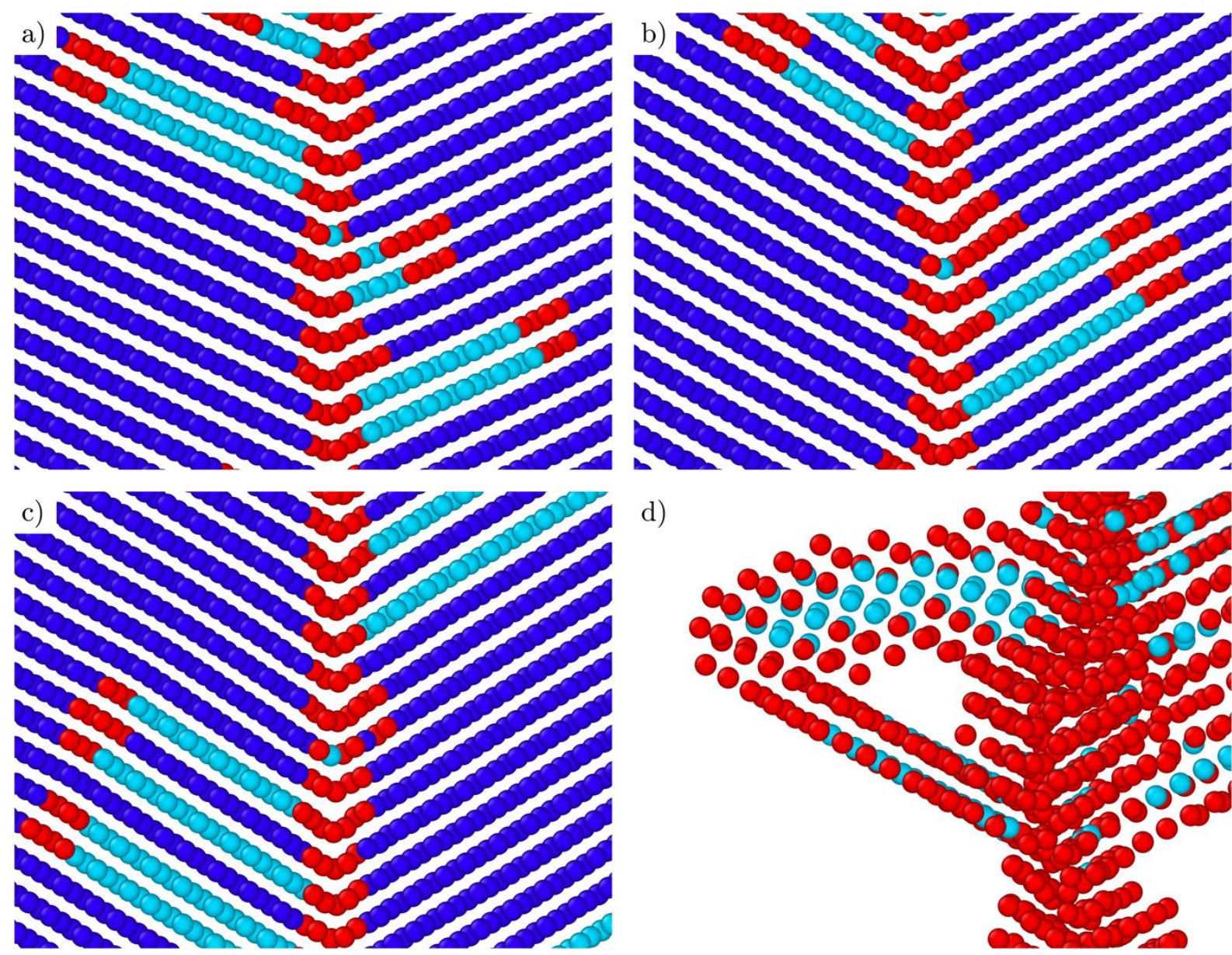

Figure 4. Representative GB dislocation nucleation responses. Atoms are colored by their CNA value, as in Figure 2; partial dislocations emerging from the GB are marked by red and turquoise atoms, identifying the leading partial and stacking faults, respectively. (а) Partial dislocation nucleation on a single slip system into each grain. (b) Dislocation nucleation on two different slip systems that share a common slip plane. The combination of the two partial dislocations leads to a full dislocation on the middle slip plane. (c) Dislocation nucleation events that exhibit both nucleation on a single slip system and nucleation on two slip systems with a common slip plane; a combination of (a) and (b). (d) Partial dislocation nucleation on two different slip systems with different slip planes. In this case, FCC atoms have been removed to more clearly show the second slip system. 

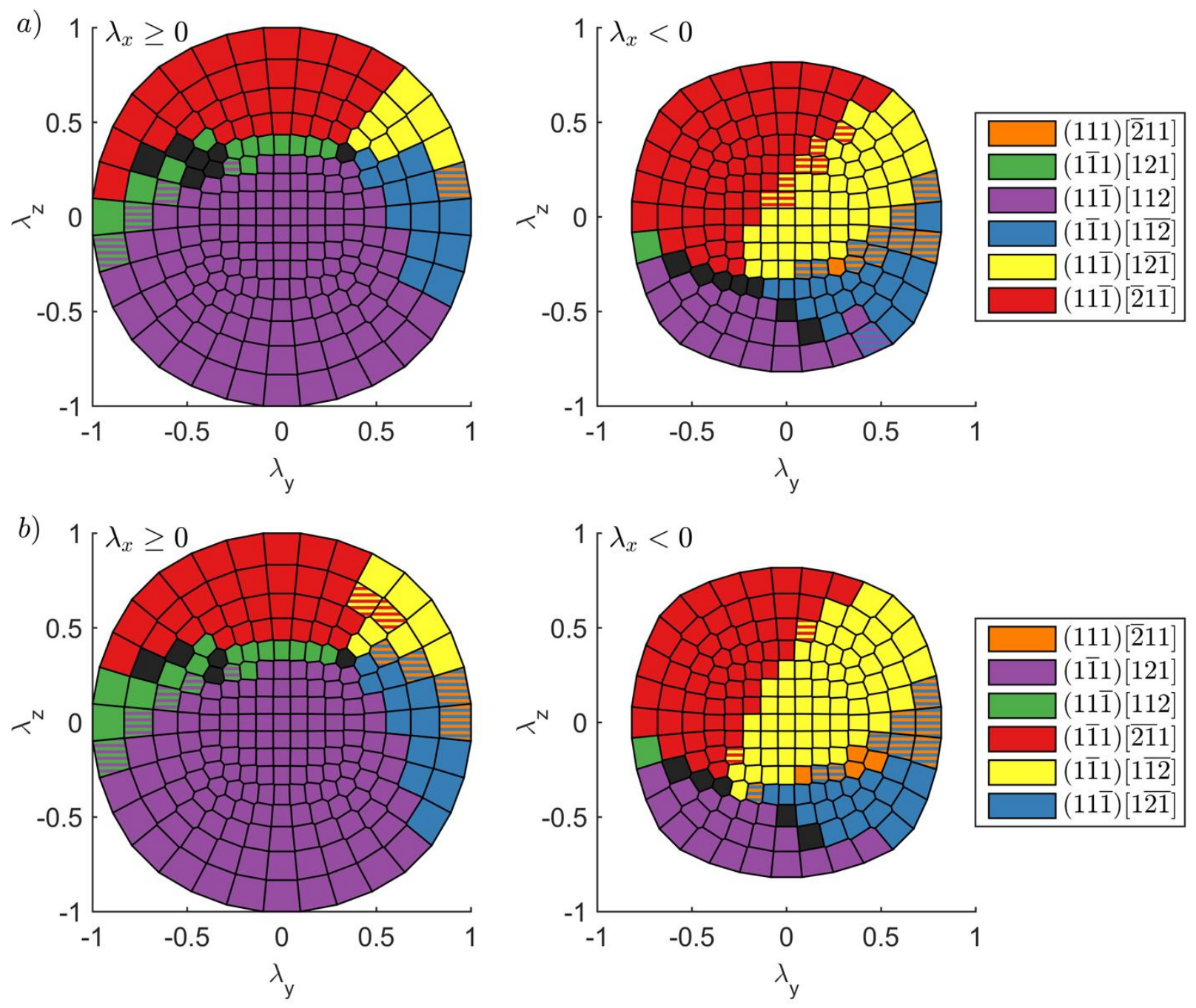

Figure 5. Schematic showing the slip systems in (a) grain A and (b) grain B, on which partial dislocation nucleation occurs for each triaxial stress state. Each voxel represents a single simulation with a particular triaxial stress state, or $\lambda$ vector. To visualize the two halves of the sphere, a stereographic projection for $\lambda_{x} \geq 0$ is shown on the left, and for $\lambda_{x}<0$ on the right. Slip systems for each grain are identified according to their respective legends. Simulations with simultaneous nucleation on multiple slip systems are thatched with both colors. 

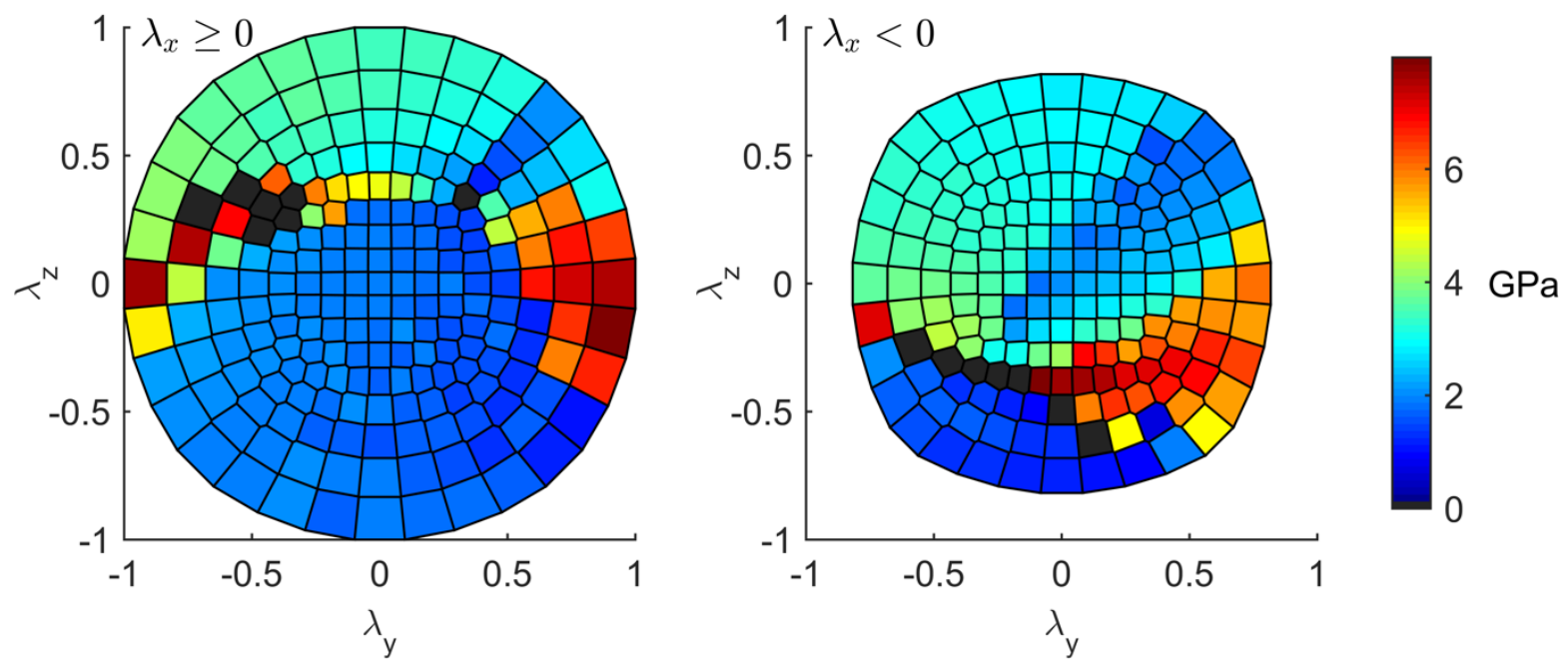

Figure 6 . The resolved shear stress $\left(\tau_{\text {rss }}\right)$ on the slip system corresponding to nucleation in grain $A$, corresponding to the slip systems shown in Figure $5 a$, for each of the triaxial stress states examined. For simulations with simultaneous nucleation on multiple slip systems, the average resolved shear stresses are used. 


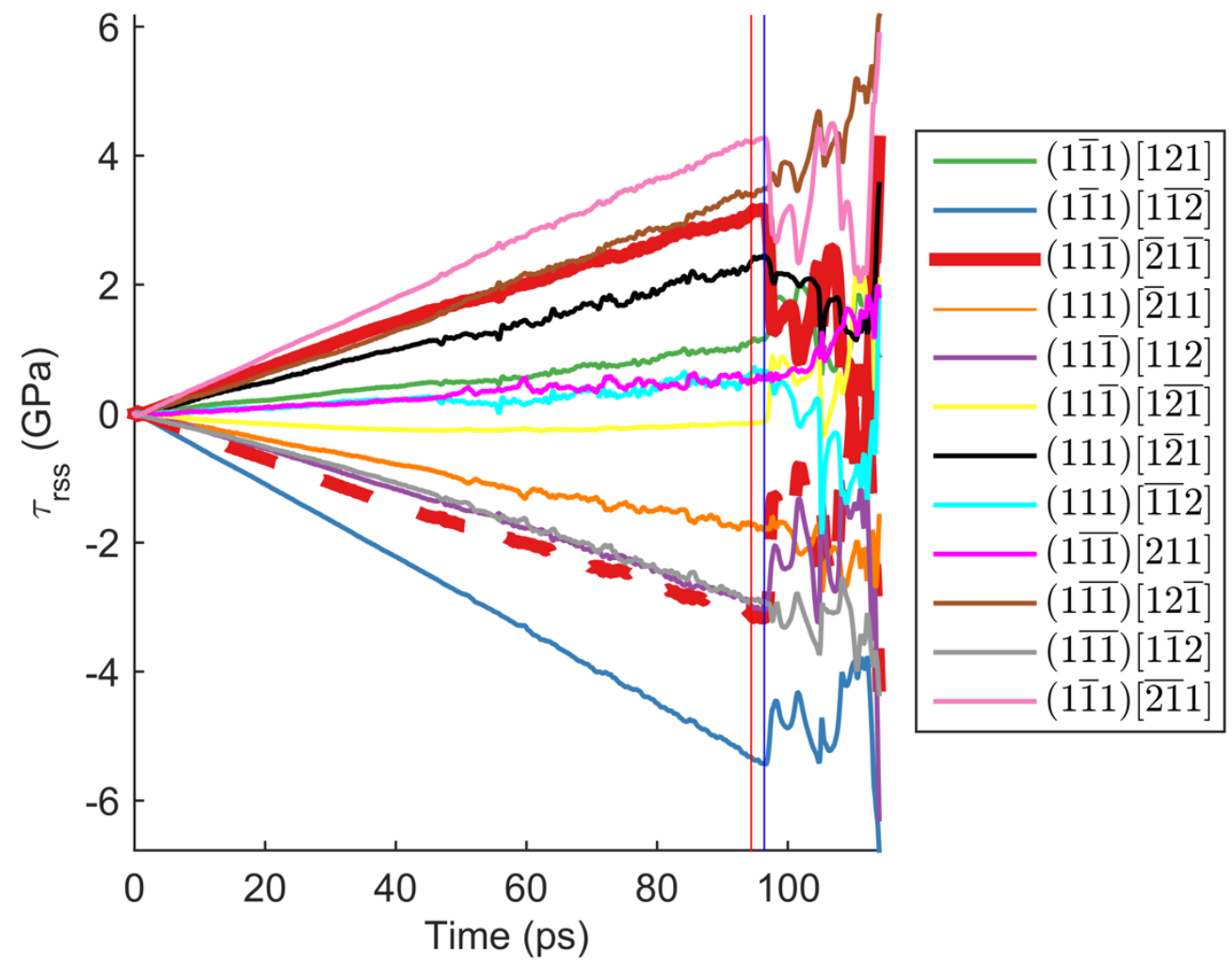

Figure 7. Resolved shear stress on each of the 12 partial slip systems in grain A as a function of time for the $\lambda=[-0.153,-0.699,0.699]$ triaxial stress state simulation. This simulation nucleates on the $(11 \overline{1})[\overline{2} 1 \overline{1}]$ slip system, which is shown as a heavy red line. For comparison with slip systems that have negative stresses, the negative of this resolved shear stress is shown as a heavy red dashed line. Note that three other slip systems have higher magnitudes of resolved shear stress than the system on which nucleation occurs. The vertical red and blue lines are again used to identify the time of the maximum stress and sampling time, respectively, as in Figure $3 b$. 

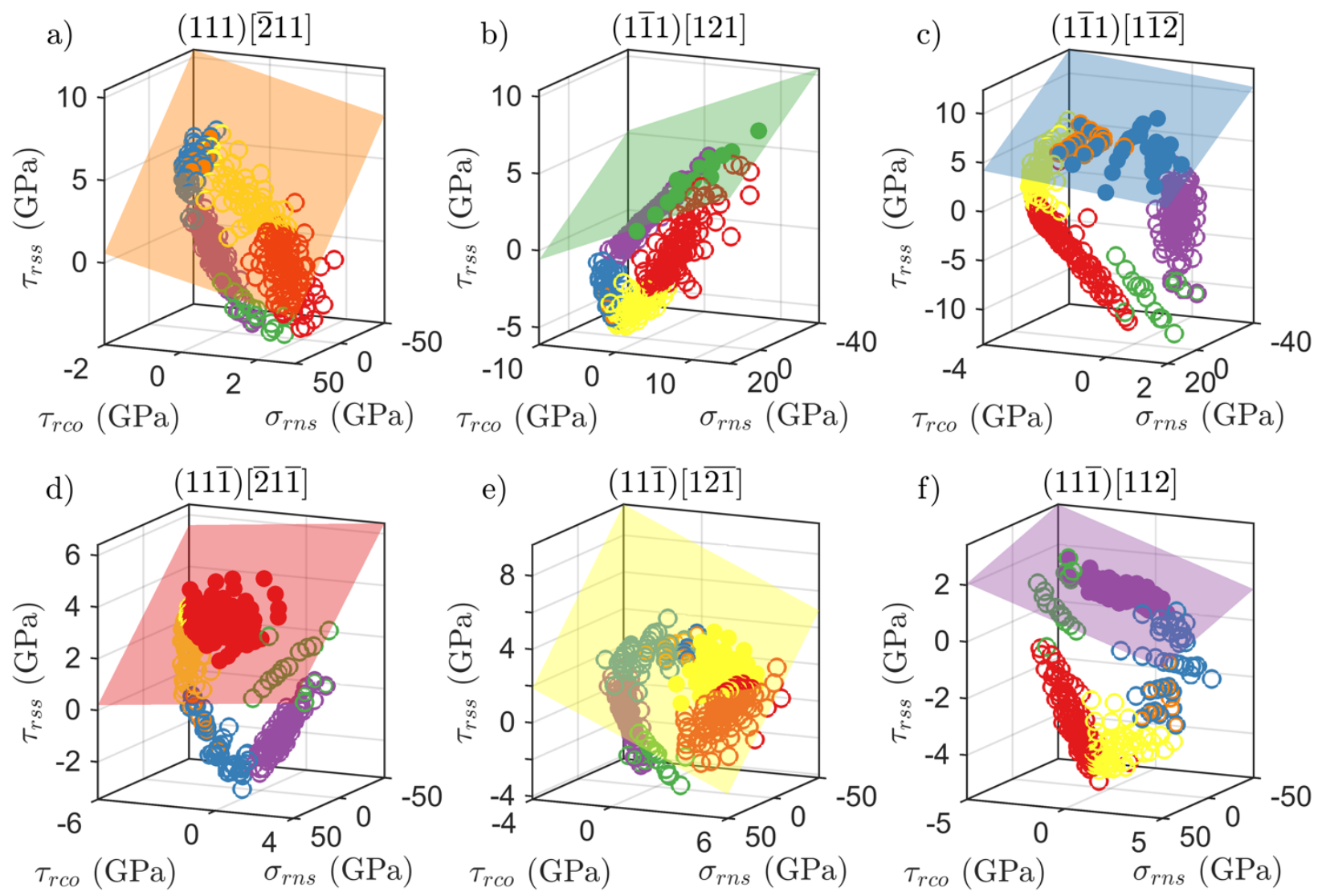

Figure 8. Plots of $\tau_{\text {rss }}, \sigma_{\text {rns }}$ and $\tau_{\text {rco }}$ for all 372 simulations, resolved onto six different slip systems (a-f) in grain A at the point of nucleation, regardless of whether nucleation occurs on that slip system. The marker colors identify the nucleation slip system for any given simulation, as defined in Fig. 5. Solid markers identify simulations where nucleation occurs on the slip system associated with that plot; open markers identify simulations where nucleation occurs on a different slip system. The plane is fit to the solid markers for each slip system and represents a criterion for GB dislocation nucleation on that particular slip system. Note that the planar fits to the closed markers rarely have open markers higher than the fitted plane. 

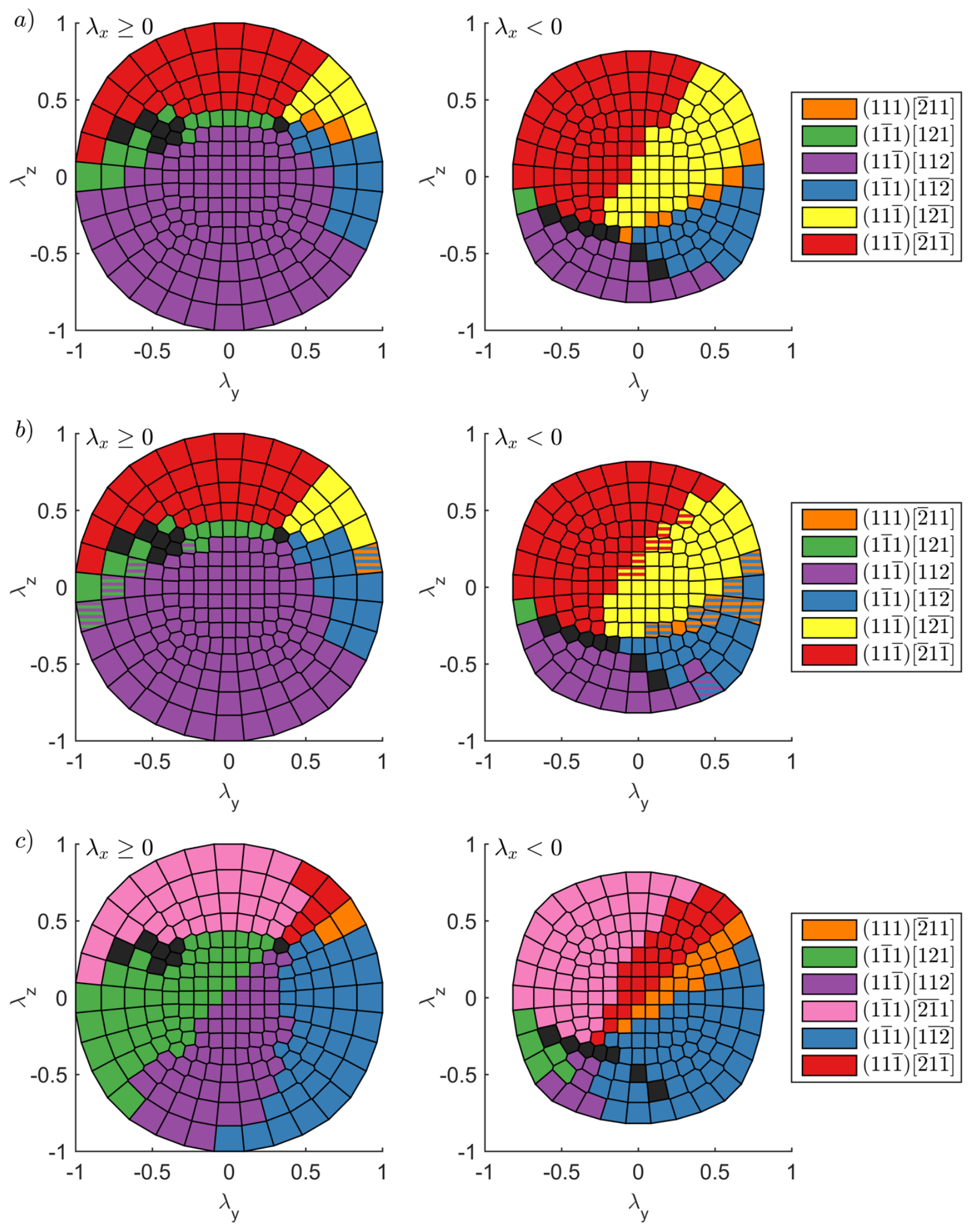
Figure 9. A comparison of the nucleated slip system for grain A (a) predicted by Eq. 3, (b) the nucleation slip system observed in the simulations (reproduction of Figure 5a), and (c) the nucleation slip system predicted by the Schmid factor. 

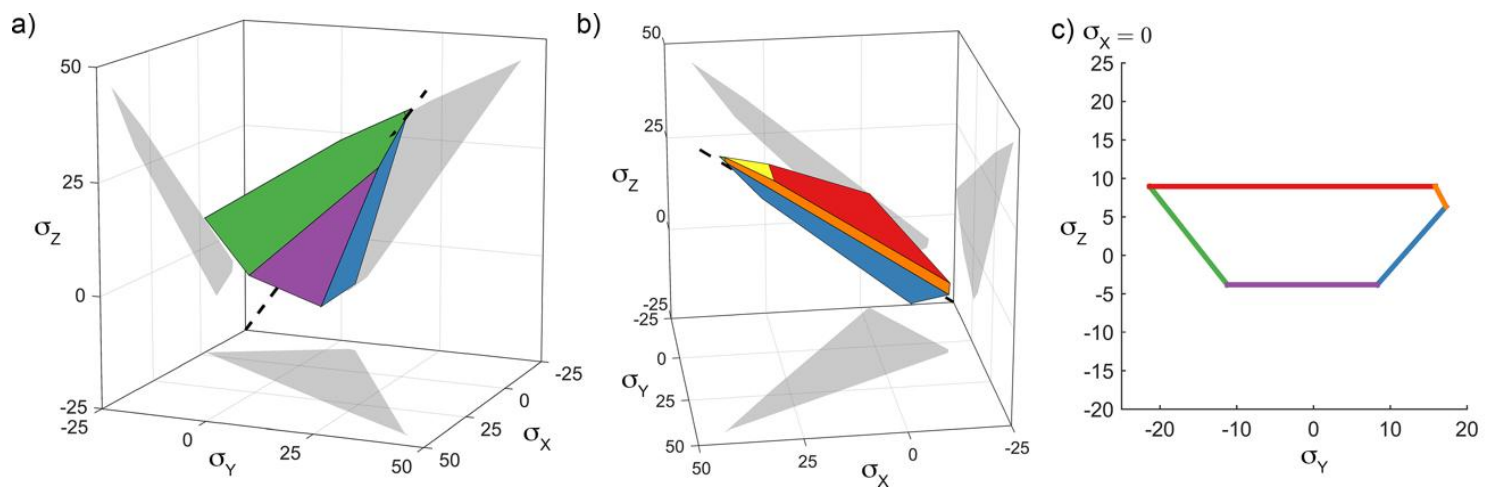

Figure 10. Two views, (a) and (b), of the theoretical nucleation surface constructed using the nucleation criteria defined by Eq. 3. Each facet of the nucleation surface corresponds to nucleation on a different slip system, colored by the same scheme as in Figure 5a. Shadow plots of the nucleation surface are projected onto the back walls of the figure for the three orthogonal views. The dashed line represents hydrostatic stress. Note that unlike a traditional plasticity envelope, the reference frame is not the principal stress space, but rather the coordinate system used in the simulations, whose orientation is not invariant. (c) Biaxial slice of the nucleation surface at $\sigma_{x}=0$. 


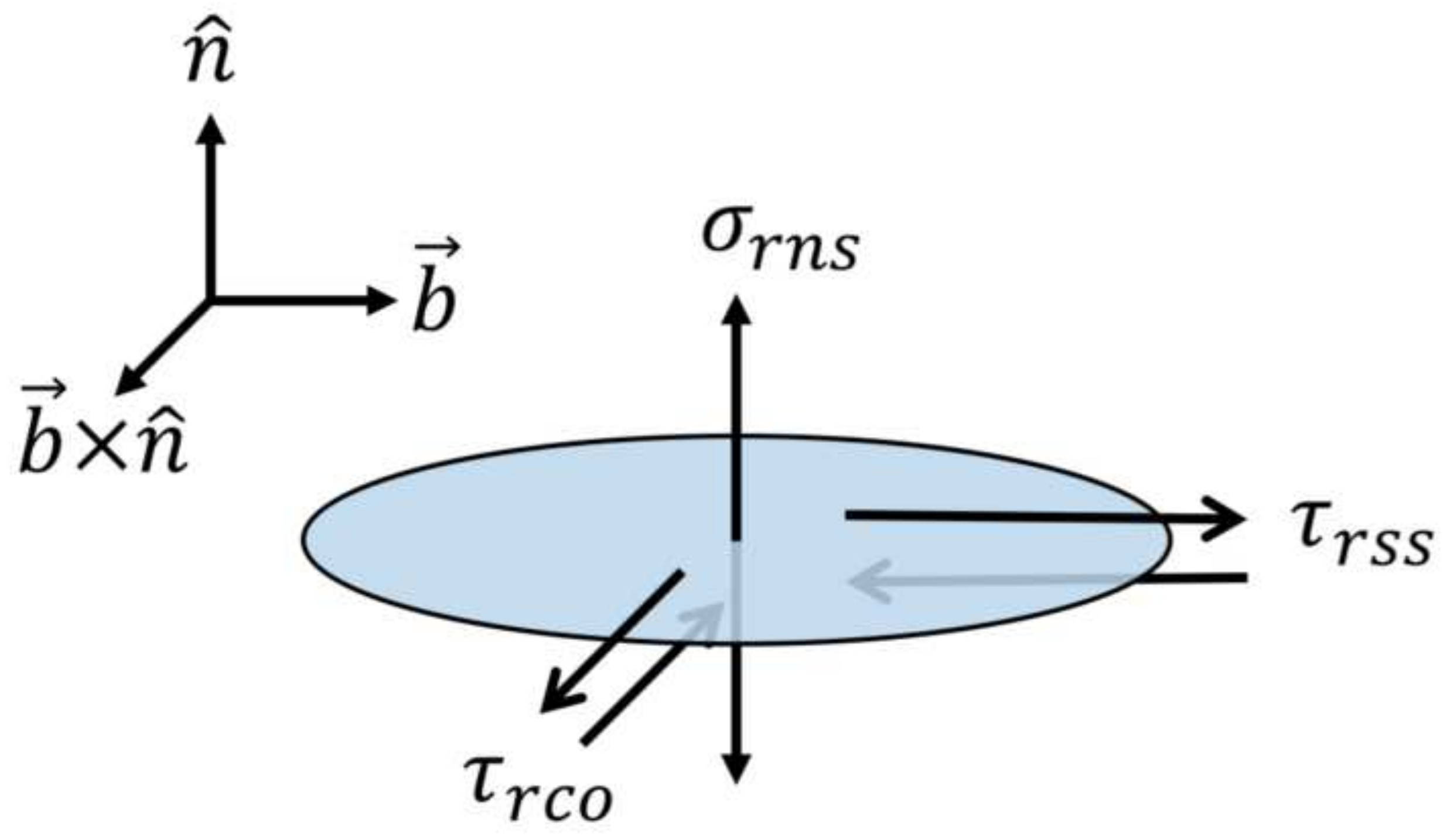

Figure 1

$\tau_{r S S}$ 
Figure 2

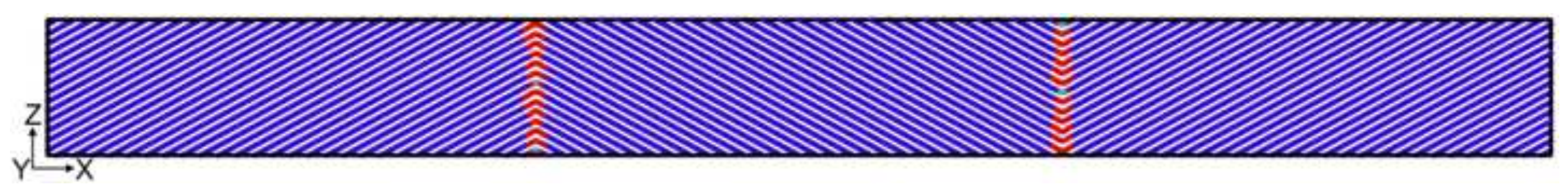



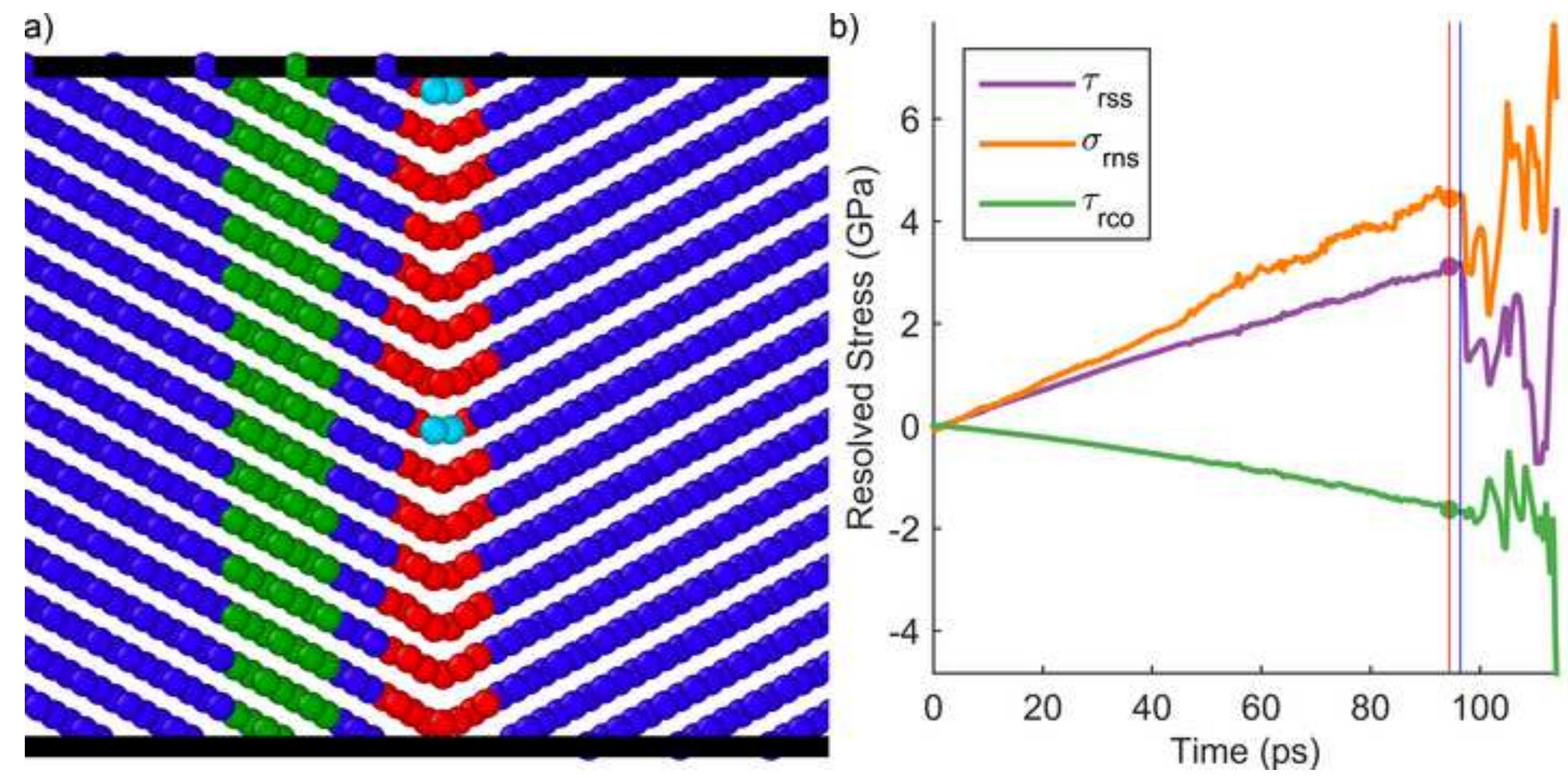

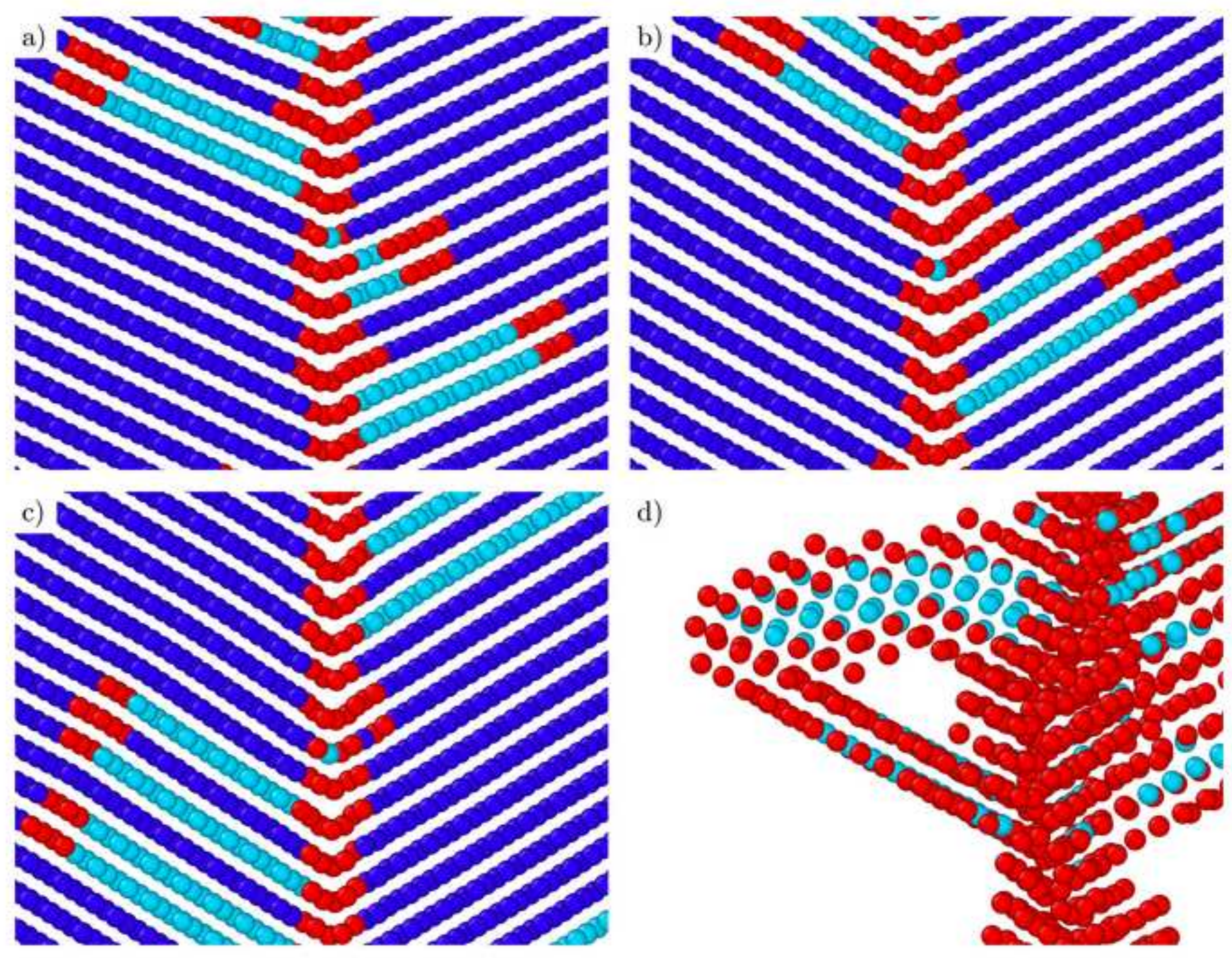

d)

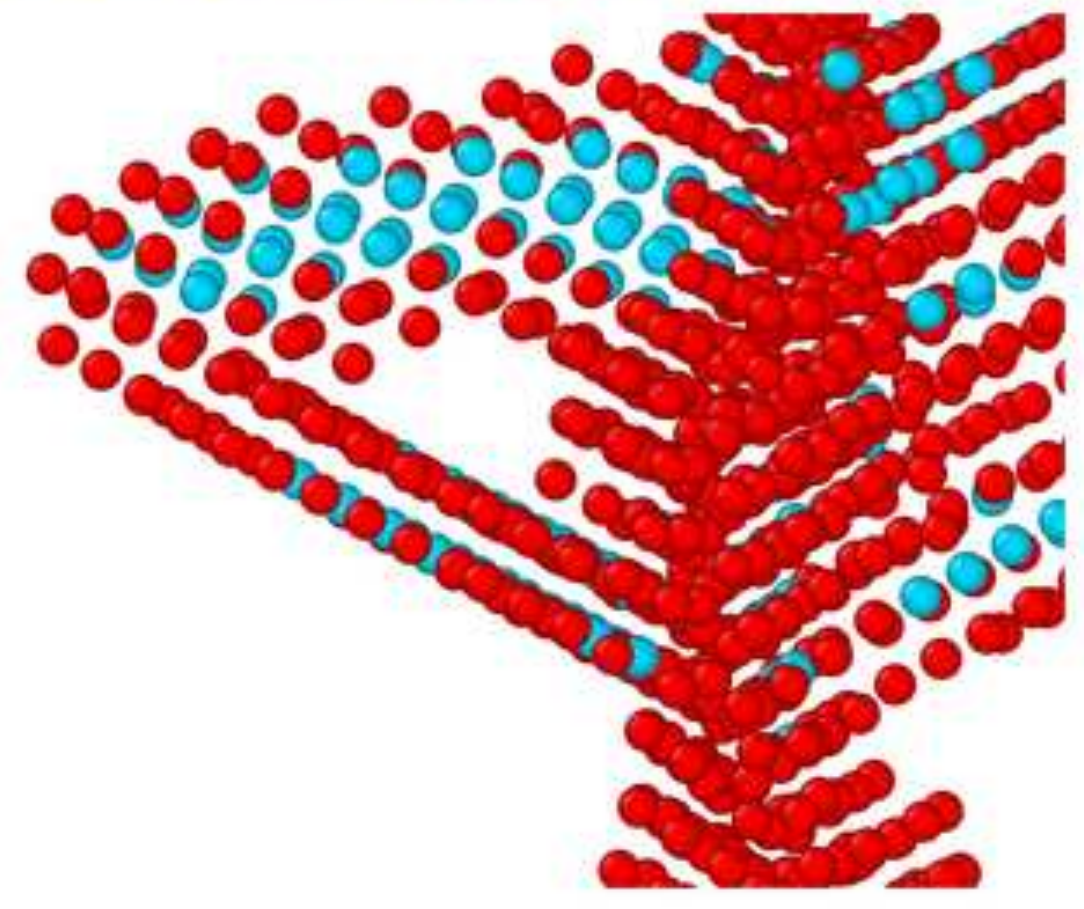



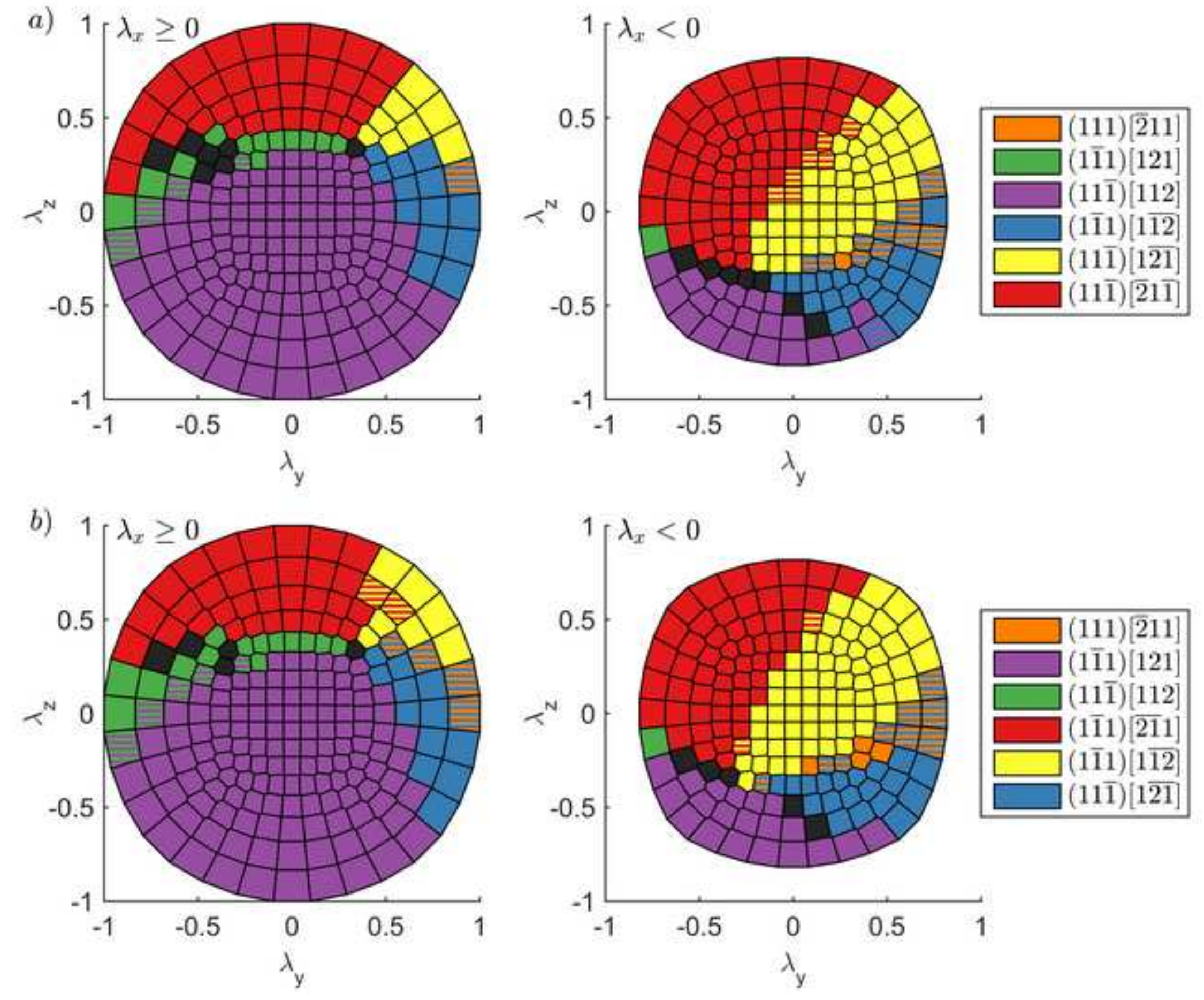

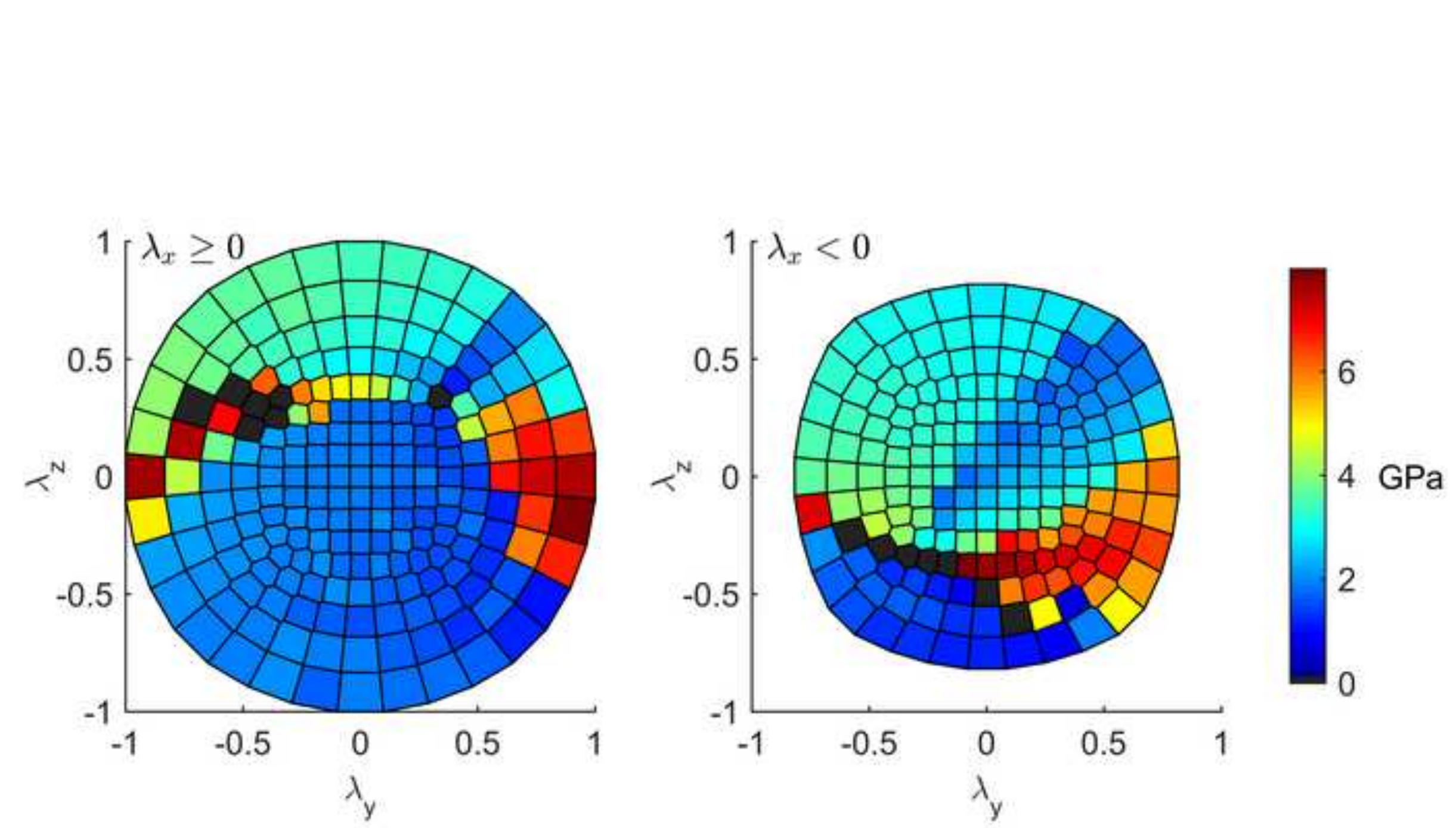


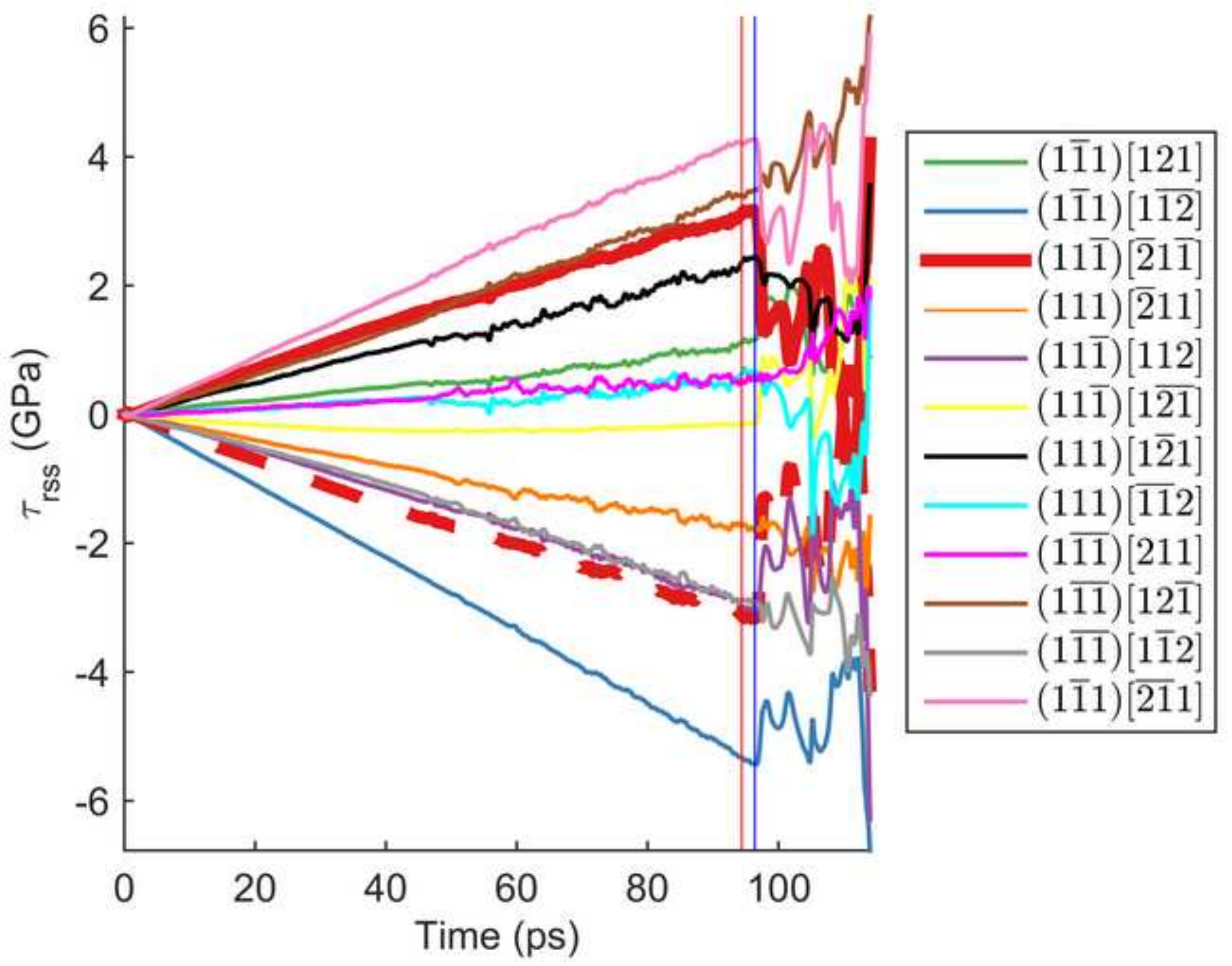



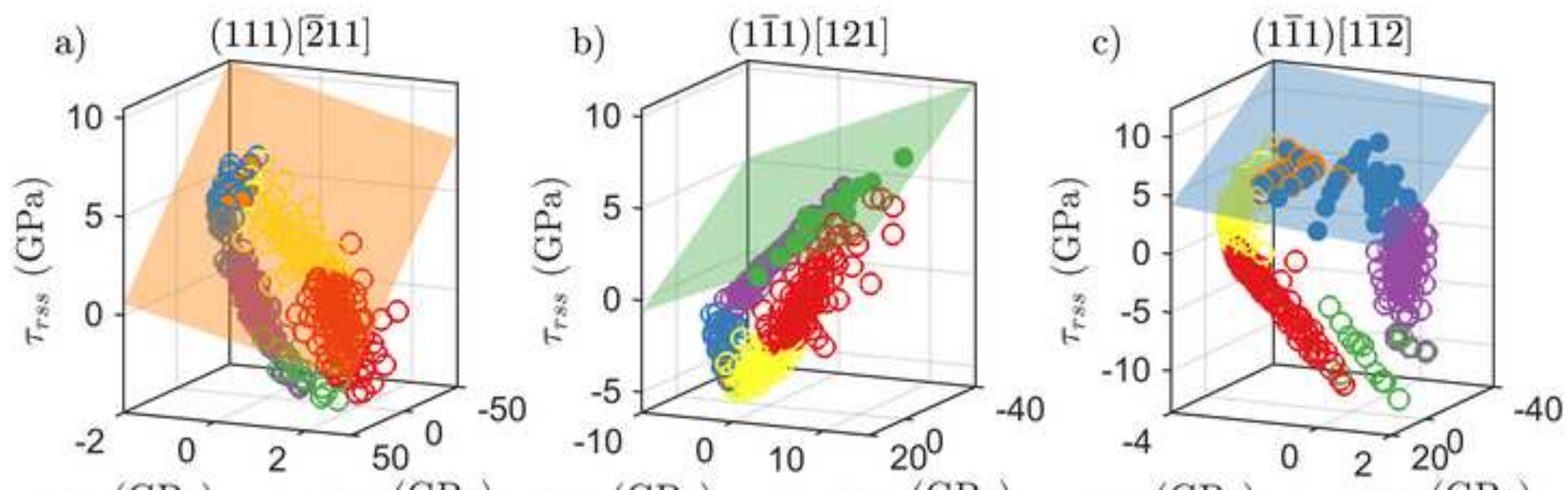

$\tau_{\text {rco }}(\mathrm{GPa})$
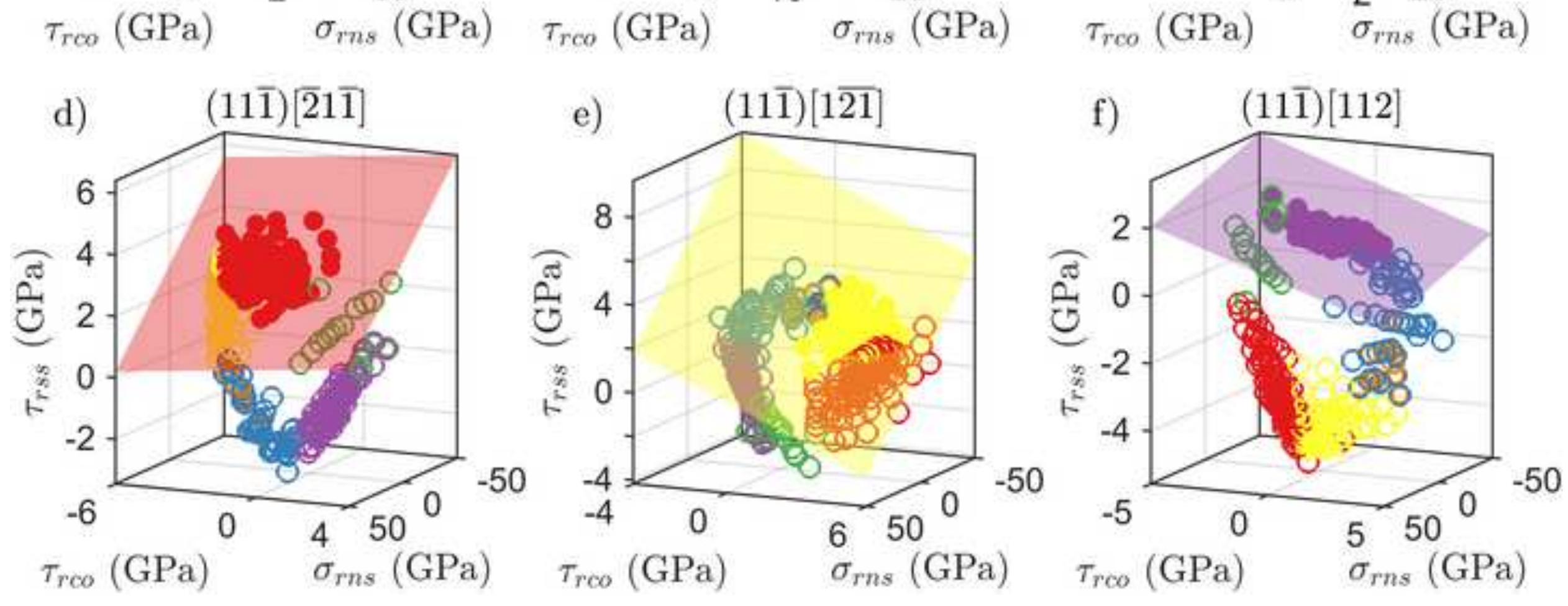

$\tau_{\text {rco }}(\mathrm{GPa})$

$\sigma_{r n s}(\mathrm{GPa}) \quad \tau_{r c o}(\mathrm{GPa})$

$\sigma_{\text {rns }}(\mathrm{GPa})$ 

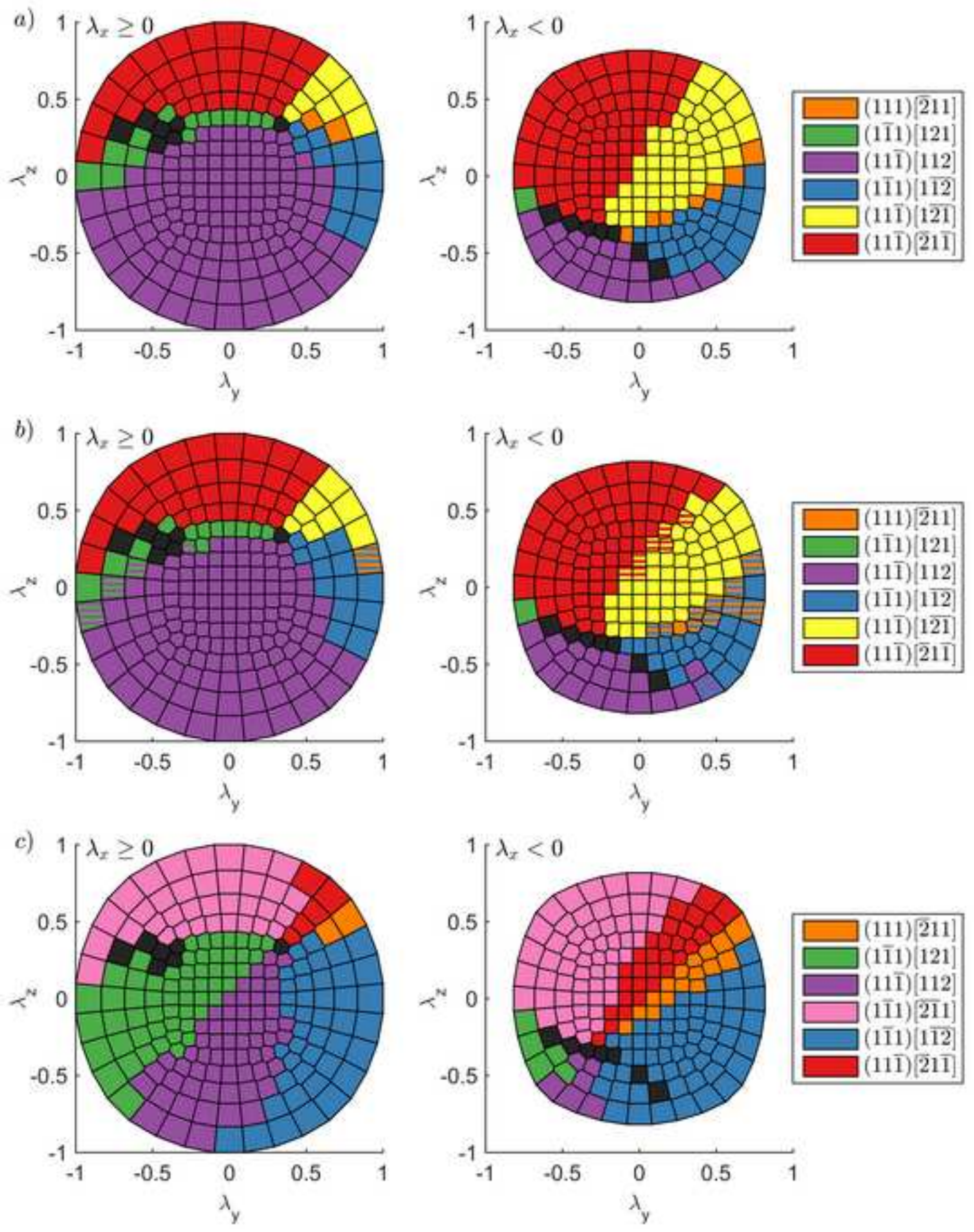

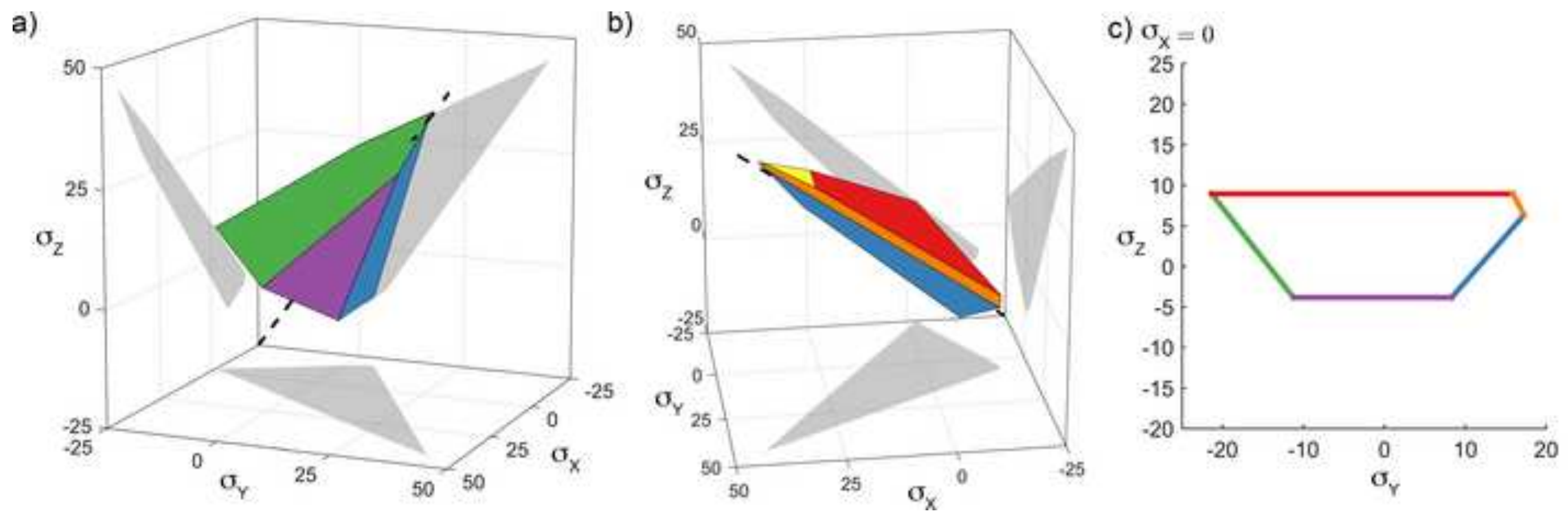
Triaxial Stress
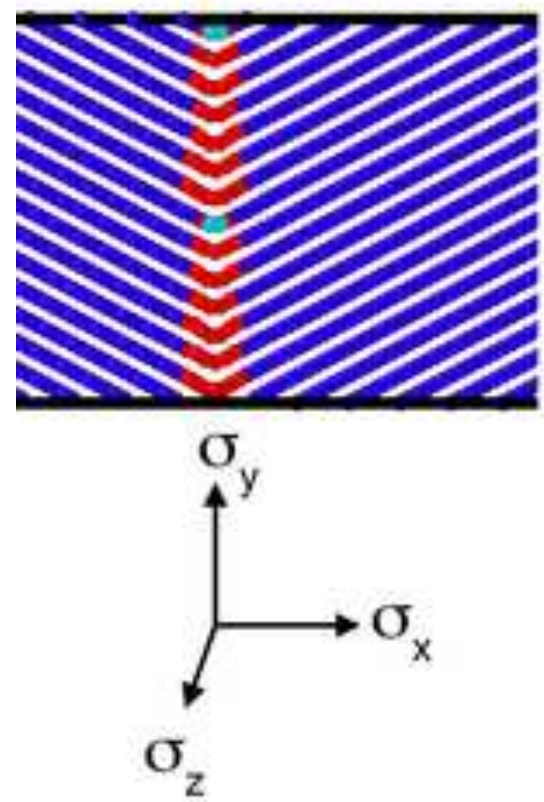

Dislocation Nucleation

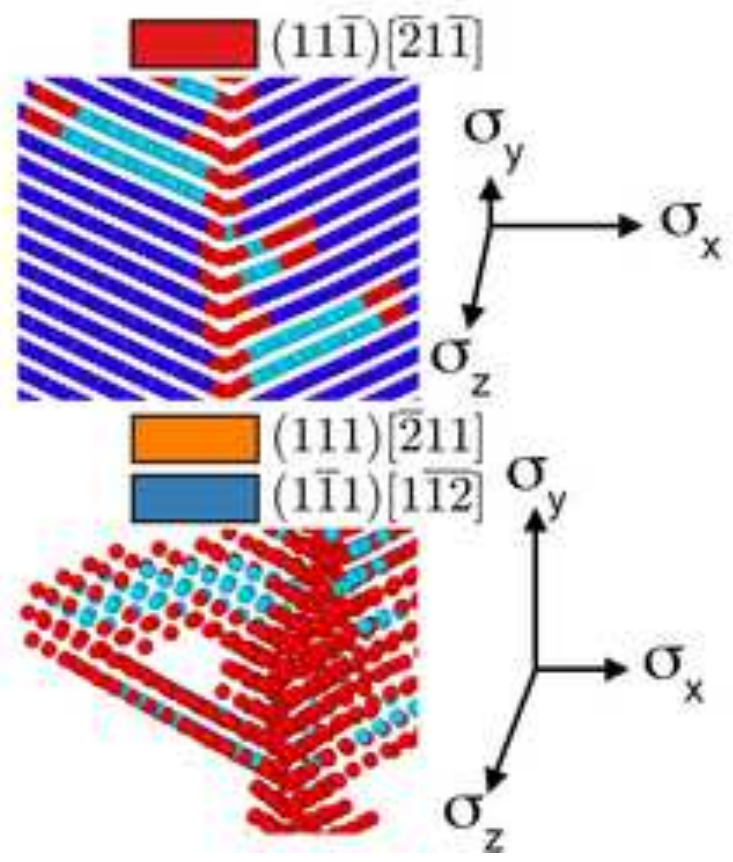

Nucleation Slip System
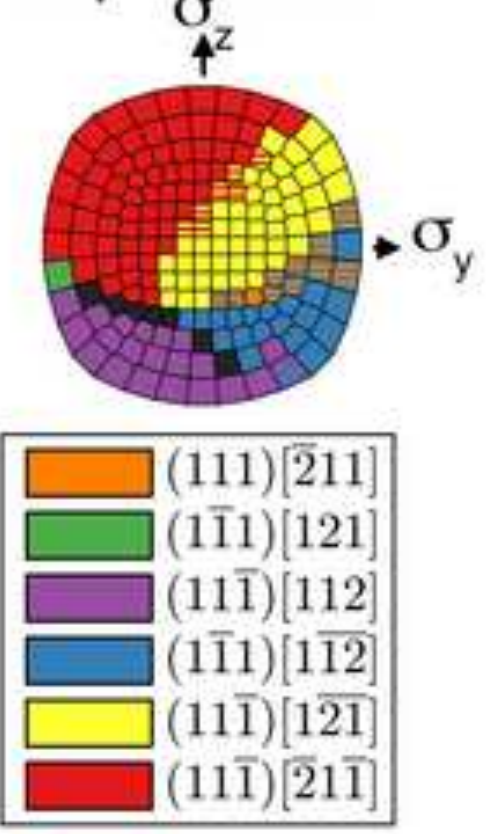

Nucleation Criteria

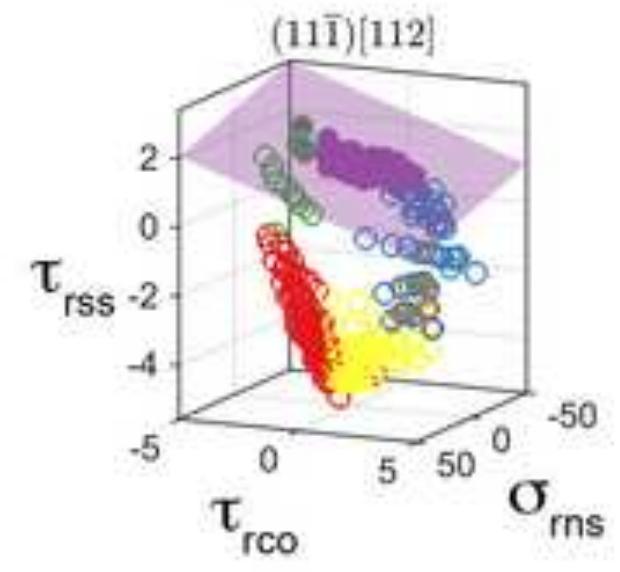

$$
1 \leq \frac{\tau_{r s s}}{\tau_{c r s s}}+\frac{\sigma_{r n s}}{\sigma_{c r n s}}+\frac{\tau_{r c o}}{\tau_{c r c o}}
$$

